\title{
The impact of episodic outflow feedback on stellar multiplicity and the star formation efficiency
}

\author{
P. F. Rohde ${ }^{\circledR},{ }^{1 \star}$ S. Walch, ${ }^{1}$ S. D. Clarke ${ }^{\circledR}, 1$ D. Seifried ${ }^{\circledR},{ }^{1}$ A. P. Whitworth ${ }^{2}$ and A. Klepitko ${ }^{1}$ \\ ${ }^{1}$ I. Physikalisches Institut, Universität zu Köln, Zülpicher Str. 77, D-50937 Köln, Germany \\ ${ }^{2}$ School of Physics and Astronomy, Cardiff University, Cardiff CF24 3AA, UK
}

Accepted 2020 September 21. Received 2020 September 2; in original form 2020 July 10

\begin{abstract}
The accretion of material on to young protostars is accompanied by the launching of outflows. Observations show that accretion, and therefore also outflows, are episodic. However, the effects of episodic outflow feedback on the core scale are not well understood. We have performed 88 smoothed particle hydrodynamic simulations of turbulent dense $1 \mathrm{M}_{\odot}$ cores to study the influence of episodic outflow feedback on the stellar multiplicity and the star formation efficiency (SFE). Protostars are represented by sink particles, which use a subgrid model to capture stellar evolution, inner-disc evolution, episodic accretion, and the launching of outflows. By comparing simulations with and without episodic outflow feedback, we show that simulations with outflow feedback reproduce the binary statistics of young stellar populations, including the relative proportions of singles, binaries, triples, etc. and the high incidence of twin binaries with $q \geq 0.95$; simulations without outflow feedback do not. Entrainment factors (the ratio between total outflowing mass and initially ejected mass) are typically $\sim 7 \pm 2$, but can be much higher if the total mass of stars formed in a core is low and/or outflow episodes are infrequent. By decreasing both the mean mass of the stars formed and the number of stars formed, outflow feedback reduces the SFE by about a factor of 2 (as compared with simulations that do not include outflow feedback).
\end{abstract}

Key words: methods: numerical-binaries: general-stars: formation-stars: low-mass-stars: protostars-stars: winds, outflows.

\section{INTRODUCTION}

One of the fundamental open questions in modern astrophysics is why molecular gas is very inefficiently converted into stars. On molecular cloud scales, the star formation efficiency (SFE) is only a few per cent (Leroy et al. 2008; Utomo et al. 2018; Schruba, Kruijssen \& Leroy 2019), and stellar feedback is presumed to be the reason for this low efficiency (Murray 2011). Along with stellar winds, ionizing radiation and supernovae, protostellar outflows are one of the feedback mechanisms that might substantially reduce the overall SFE, particularly in regions where there are no massive stars (e.g. Nakamura \& Li 2007; Hansen et al. 2012; Federrath et al. 2014; Krumholz et al. 2014; Cunningham et al. 2018; Li, Klein \& McKee 2018)

Low-mass stars form preferentially in pre-stellar cores, which tend to be concentrated in dense filaments inside molecular clouds (Shu \& Adams 1987; André et al. 2007; Myers 2009; André et al. 2014; Könyves et al. 2015; Marsh et al. 2016; Könyves et al. 2020). In contrast to the elongated shapes of filaments, pre-stellar cores are approximately spherical, and their density profiles are often described as Bonnor-Ebert spheres, with typical radii of $R_{\mathrm{CORE}} \sim 0.01 \mathrm{pc}$ to 0.1 pc (Bonnor 1956; Ebert 1957; Johnstone et al. 2000; Alves, Lada \& Lada 2001; Tafalla et al. 2004; Könyves et al. 2020). The pre-stellar core mass function (CMF) approximates to a lognormal distribution

^E-mail: rohde@ph1.uni-koeln.de with a peak around $\sim 0.5 \mathrm{M}_{\odot}$ (Könyves et al. 2015, 2020; Marsh et al. 2016). Molecular line observations of pre-stellar cores show nonthermal velocity components indicating internal turbulence (André et al. 2007; Pineda et al. 2011; Friesen et al. 2017).

Protostellar outflows often accompany the star formation process (Bally 2016). Observations and numerical simulations suggest that outflows consist of two components: a collimated high-velocity jet (Mundt \& Fried 1983; Reipurth \& Bally 2001; Tafalla et al. 2010; Lee et al. 2017) and a slower wide-angle disc wind, launched further out in the accretion disc (Machida 2014; Tabone et al. 2017; Liu et al. 2018; Louvet et al. 2018; Zhang et al. 2019). Both components are known to be rotating (Hirota et al. 2017; Lee et al. 2017; Zhang et al. 2018, 2019). Therefore, outflows carry away angular momentum from the disc-star system, which in turn allows the central protostar to accrete while staying below its breakup speed (Pudritz et al. 2007; Bjerkeli et al. 2016).

Protostellar jets are launched from the innermost regions of protostellar accretion discs. Numerous authors have simulated protostellar outflows self-consistently using magnetohydrodynamic (MHD) simulations (e.g. Machida, Inutsuka \& Matsumoto 2009; Hennebelle et al. 2011; Price, Tricco \& Bate 2012; Seifried et al. 2012; Machida \& Hosokawa 2013; Bate, Tricco \& Price 2014; Machida 2014; Tomida 2014; Tomida, Okuzumi \& Machida 2015; Lewis \& Bate 2017; Machida \& Basu 2019; Saiki \& Machida 2020). However, such simulations must resolve the launching region down to $r_{\mathrm{LAUNCH}} \sim \mathrm{R}_{\odot}$ to reproduce the extremely high-velocity jet component that originates in the innermost disc region. It is 
presently not computationally feasible to follow the evolution of protostars through the whole protostellar phase using such a high resolution. Other authors therefore mitigate this problem by invoking almost resolution-independent subgrid models to launch outflows (Nakamura \& Li 2007; Cunningham et al. 2011; Federrath et al. 2014; Myers et al. 2014; Offner \& Arce 2014; Peters et al. 2014; Kuiper, Yorke \& Turner 2015; Offner \& Chaban 2017; Li et al. 2018; Rohde et al. 2019)

Exactly how the gas is launched is still not well understood (see, e.g. the reviews of Arce et al. 2007; Frank et al. 2014; Bally 2016). However, the consensus is that outflows are accretion powered: gravitational energy is converted into kinetic and magnetic energy, which then drives and collimates the outflow, either through magnetic pressure or magneto-centrifugal forces (Blandford \& Payne 1982; Königl \& Pudritz 2000; Lynden-Bell 2003; Pudritz et al. 2007; Machida, Inutsuka \& Matsumoto 2008; Seifried et al. 2012). Since the accretion on to a protostar is episodic, outflows are also episodic (Reipurth 1989; Hartigan, Edwards \& Ghandour 1995; Hartmann 1997; Königl \& Pudritz 2000; Arce et al. 2007; Hennebelle et al. 2011; Kuiper et al. 2015; Bally 2016; Choi et al. 2017; Cesaroni et al. 2018; Samal et al. 2018; Zhang et al. 2019).

Protostellar outflows inject a significant amount of energy and momentum into the surroundings (Arce et al. 2010; Plunkett et al. 2013; Feddersen et al. 2020), and are likely to have a profound impact on their host cores. This is especially true in the context of low-mass star formation where other feedback mechanisms do not come into play. The 'primary' ejected gas from the immediate vicinity of the protostar entrains 'secondary' core material, thereby carving out a cavity which widens over time (Arce \& Sargent 2006). Within the cavity, accretion flows on to the protostar are suppressed, lowering the amount of gas which can fall directly on to the protostar, and hence lowering the protostellar accretion rate. (Wang et al. 2010). The resulting feedback loop of accretion and outflow launching is not fully understood. Because outflows act to disperse a star's birth core, they are presumed to play a role in terminating the accretion process (Zhang et al. 2016). Theoretical studies show that this may cause the SFE on core scales to be as low as 15-50 per cent (Machida \& Hosokawa 2013; Offner \& Arce 2014; Offner \& Chaban 2017). However, more observations and theoretical studies are needed to fully understand the effects of outflow feedback on core scales.

The stellar initial mass function (IMF; Kroupa 2002; Chabrier 2003) and the initial statistics of multiple systems (e.g. Duchêne \& Kraus 2013) are key constraints on theories of star formation. Raghavan et al. (2010) find that in the field roughly 50 per cent of systems are single stars like our Sun; all the rest are binaries or higher-order multiples (i.e. triples, quadruples, quintuples, etc., hereafter HOMs). Recent observations have started to reveal the multiplicity statistics of pre-main-sequence stars (Duchêne et al. 2007; Connelley, Reipurth \& Tokunaga 2008; Chen et al. 2013; Pineda et al. 2015; Tobin et al. 2016; Shan et al. 2017; Duchêne et al. 2018; Tobin et al. 2018; Kounkel et al. 2019). Tobin et al. (2016) have observed the Perseus molecular cloud using the VLA and report an overall multiplicity fraction of $\mathrm{mf}=0.4$ for Class $0 / \mathrm{I}$ protostars. Like Chen et al. (2013), they find that $\mathrm{mf}$ decreases for later evolutionary stages. Dynamical $N$-body interactions are probably the main reason for the decay of HOMs (e.g. Bate \& Bonnell 2005; Goodwin et al. 2007). Another observed property of low-mass stellar multiples is the excess of almost equal-mass binary systems, referred to as 'twin' binaries (Lucy 2006; Simon \& Obbie 2009; Kounkel et al. 2019).

Although multiplicity statistics are an important benchmark for simulations of star formation, such simulations should also reveal the detailed physical processes that deliver the observed multiplicity statistics (see, e.g. Offner 2011; Bate 2012; Lomax et al. 2015; Li et al. 2018; Kuffmeier, Calcutt \& Kristensen 2019; Wurster, Bate \& Price 2019). Here we explore the effect of outflow feedback on the formation and evolution of multiple systems.

The paper is structured as follows. In Section 2, we describe the computational method, outline modifications to the subgrid outflow model developed earlier by Rohde et al. (2019), and define the initial and boundary conditions. In Section 3, we present the results of the simulations and discuss how the stellar properties depend on the initial conditions. In Section 4, we describe the multiplicity statistics and how they are influenced by outflow feedback. In Section 5, we analyse the properties of the outflows and their relation to the SFE. In Section 6 we summarize our results.

\section{COMPUTATIONAL METHOD}

\subsection{SPH code GANDALF}

For the hydrodynamical simulations, we use the highly objectorientated smoothed particle hydrodynamics (SPH) and mesh-less finite-volume (MFV) code GANDALF (Hubber, Rosotti \& Booth 2018). GANDALF adopts the 'grad-h' SPH formulation (Springel \& Hernquist 2002) with an M4 kernel (Monaghan \& Lattanzio 1985) and $\eta=1.2$, giving on average $\sim 58$ neighbours. GANDALF uses hierarchical block time-stepping. In our simulations the number of allowed time-step levels is $N_{\mathrm{LVL}}=9$. Therefore an SPH particle on the highest level has $2^{N_{\mathrm{LVL}}}=512$ times more time-steps than a particle on the lowest level. During a time-step, all particles are allowed to adapt to higher levels if this is necessary. GANDALF uses the artificial viscosity prescription proposed by Morris \& Monaghan (1997), regulated by a time-dependent switch (Cullen \& Dehnen 2010). GANDALF offers various integration schemes, and we choose the second-order Leapfrog KDK scheme.

As in Rohde et al. (2019), we use the approximate radiative heating and cooling algorithm of Stamatellos et al. (2007). This method uses local SPH particle quantities to estimate a mean optical depth, which is then used to compute heating and cooling rates. The method accounts for changes in specific heat due to dissociation and ionization of $\mathrm{H}$ and $\mathrm{He}$. The opacity accounts for ice-mantle evaporation and dust sublimation, as well as the switch from dust opacity to molecular-line opacity. In contrast to Stamatellos et al. (2007), we do not use the local gravitational potential, but the local pressure gradient, to estimate the mean optical depth. This change to the original method has been proposed by Lombardi, McInally \& Faber (2015), and improves the behaviour in nonspherical geometries, such as accretion discs and collision interfaces.

\subsection{Sink particles}

Sink particles, as originally proposed by Bate, Bonnell \& Price (1995), are used in pre-stellar core-collapse simulations to limit the otherwise continuously decreasing time-steps. We use the improved sink particle description introduced by Hubber, Walch \& Whitworth (2013). Sink particles have radius $R_{\text {SINK }} \sim 1 \mathrm{AU}$, and are introduced at densities exceeding $\rho_{\text {SINK }}=10^{-10} \mathrm{~g} \mathrm{~cm}^{-3}$. We use gravitational softening on scales of order $R_{\text {SINK }}$ to make the $N$-body integration more robust. SPH particles in the vicinity of a sink particle are not accreted instantaneously, but smoothly over a few time-steps. Therefore the vicinity of a sink particle is not empty, and this leads to improved hydrodynamical behaviour. Besides limiting the timesteps, sink particles serve as active star particles, each hosting the four subgrid models detailed in the next four subsections. 


\subsection{Episodic accretion}

Following Stamatellos, Whitworth \& Hubber (2012) we divide sink particles into an unresolved inner accretion disc (IAD) and a central protostar. We keep track of the masses, $M_{\mathrm{IAD}}$ and $M_{\star}$, and the angular momenta, $\boldsymbol{L}_{\mathrm{IAD}}$ and $\boldsymbol{L}_{\star}$, of the inner accretion disc and the central protostar,

$M_{\mathrm{SINK}}=M_{\mathrm{IAD}}+M_{\star}$,

$\boldsymbol{L}_{\mathrm{SINK}}=\boldsymbol{L}_{\mathrm{IAD}}+\boldsymbol{L}_{\star}$.

Gas accreted by the sink particle is initially stored in the IAD. This gas may then be accreted on to the central protostar via two accretion channels,

$$
\frac{\mathrm{d} M_{\star}}{\mathrm{d} t}=\left.\frac{\mathrm{d} M}{\mathrm{~d} t}\right|_{\mathrm{BG}}+\left.\frac{\mathrm{d} M}{\mathrm{~d} t}\right|_{\mathrm{MRI}} .
$$

The background accretion rate, $\left.\frac{\mathrm{d} M}{\mathrm{~d} t}\right|_{\mathrm{BG}}=10^{-7} \mathrm{M}_{\odot} \mathrm{yr}^{-1}$, allows for low but continuous accretion of gas on to the central protostar. The additional episodic accretion rate is much higher, on average $\left.\frac{\mathrm{d} M}{\mathrm{~d} t}\right|_{\mathrm{MRI}} \simeq 5 \times 10^{-4} \mathrm{M}_{\odot} \mathrm{yr}^{-1}$, but only contributes during outburst events, which typically last a few tens of years. Stamatellos et al. (2012) assume that a combination of gravitational and magnetorotational instabilities (MRI) acts as the main trigger for outbursts (Zhu, Hartmann \& Gammie 2009; Zhu et al. 2010). In this way we obtain realistic accretion rates, similar to those observed in FU Orionis Type stars (Bell \& Lin 1994). We use the episodic accretion rate for the following subgrid models. Varying $\left.\frac{\mathrm{d} M}{\mathrm{~d} t}\right|_{\text {MRI }}$ has little effect on the outcome of the simulations (Rohde et al. 2019).

\subsection{Stellar evolution model}

Improving upon Rohde et al. (2019) we implement the one-zone stellar evolution model described in Offner et al. (2009), originally introduced by Nakano, Hasegawa \& Norman (1995), and subsequently improved by Nakano et al. (2000) and Tan \& McKee (2004). This subgrid model describes the evolution of the stellar radius, $R_{\star}$, and luminosity, $L_{\star}$, due to the energy balance between accretion, gravitational contraction, nuclear burning, ionization and radiation. The change in protostellar radius, $\dot{R}_{\star}$, is given by

$$
\begin{aligned}
\dot{R}_{\star}= & \frac{2}{M_{\star}} \frac{\mathrm{d} M_{\star}}{\mathrm{d} t}\left(1-\frac{1-f_{\mathrm{K}}}{a_{\mathrm{G}}(n) \beta}+\frac{1}{2} \frac{d \log \beta}{d \log M_{\star}}\right) R_{\star} \\
& -\frac{2}{a_{\mathrm{G}}(n) \beta}\left(\frac{R_{\star}}{\mathrm{G} M_{\star}^{2}}\right)\left(L_{\mathrm{INT}}+L_{\mathrm{DI}}-L_{\mathrm{DB}}\right) R_{\star} .
\end{aligned}
$$

Here, $G$ is the gravitational constant, $f_{\mathrm{K}}=0.5$ is the fraction of kinetic energy that is radiated away in the inner accretion disc, $a_{\mathrm{G}}(n)$ is the gravitational energy coefficient for a sphere with polytropic index $n<5$ (Nakano et al. 2000, and references therein), $\beta$ is the ratio of gas pressure to total pressure (gas plus radiation) in the protostar, $L_{\mathrm{INT}}$ is the internal luminosity, $L_{\mathrm{DI}}$ is the power required to dissociate and ionize the accreted gas, and $L_{\mathrm{DB}}$ is the power released by deuterium burning.

This model follows the protostellar evolution through six distinct phases: (i) the initial 'pre-collapse' phase; (ii) the 'no burning' phase; (iii) the 'core deuterium burning at fixed $\mathrm{T}_{\mathrm{C}}$ ' phase; (iv) the 'core deuterium burning at variable $\mathrm{T}_{\mathrm{C}}$ ' phase; $(\mathrm{v})$ the 'shell deuterium burning' phase; and (vi) the 'zero-age main-sequence' phase (Tout et al. 1996).

We follow the implementation described by Offner et al. (2009) and also used by Murray, Goyal \& Chang (2018) and Cunningham et al. (2018). However, we use the mass of the subgrid protostar, $M_{\star}$ (rather than the mass of the sink particle, $M_{\mathrm{SINK}}$ ) and the episodic accretion rate from the IAD on to the protostar, $\frac{\mathrm{d} M_{\star}}{\mathrm{d} t}$ (rather than the sink particle's accretion rate, $\frac{\mathrm{d} M_{\mathrm{SINK}}}{\mathrm{d} t}$; Section 2.3). The accretion luminosity depends linearly on the accretion rate and is therefore highly variable due to the episodic nature of accretion on to the protostar.

\subsection{Radiative feedback}

Radiative feedback from young protostars can heat and stabilize their surrounding accretion discs, thus suppressing further disc fragmentation (Jones \& Bate 2018). Theoretical studies have shown that this reduces the number of brown dwarfs and low-mass protostars formed (Chabrier 2003; Offner et al. 2009; Rice et al. 2011; Guszejnov, Krumholz \& Hopkins 2016; Guszejnov, Hopkins \& Krumholz 2017). However, continuous radiative feedback (i.e. neglecting episodic accretion effects) tends to suppress the formation of brown dwarfs and low-mass protostars too efficiently, resulting in a lower stellar multiplicity than observed (Stamatellos et al. 2012; Lomax et al. 2014, 2015; Mercer \& Stamatellos 2017).

We make use of the episodic accretion model in Stamatellos et al. (2012; Section 2.3), in combination with the stellar evolution model in Offner et al. (2009; Section 2.4) to compute the highly variable protostellar luminosities. These luminosities are taken into account by invoking a pseudo background radiation field with temperature, $T_{\mathrm{BG}}$. At general position $\boldsymbol{r}, T_{\mathrm{BG}}$ is given by

$T_{\mathrm{BG}}^{4}(\boldsymbol{r})=(10 \mathrm{~K})^{4}+\sum_{n}\left(\frac{L_{\star, n}}{16 \pi \sigma_{\mathrm{SB}}\left|\boldsymbol{r}-\boldsymbol{r}_{\star, n}\right|^{2}}\right)$

(Stamatellos et al. 2007). Here, $\boldsymbol{r}_{\star, n}$ and $L_{\star, n}$ are the position and luminosity of the $n$th protostar. In the vicinity of a protostar $T_{\mathrm{BG}}$ decreases with distance $d$ from the protostar approximately as $d^{-1 / 2}$. This method will not capture accurately the radiative feedback from massive stars. However, we are interested here in the formation of low-mass stars (our initial core mass is just $1 \mathrm{M}_{\odot}$ ) and the model has been extensively tested in this regime (Stamatellos et al. 2012; Lomax et al. 2014, 2015; Mercer \& Stamatellos 2017; Rohde et al. 2019).

\subsection{Outflow feedback}

We use the subgrid episodic outflow model presented in Rohde et al. (2019) with a few modifications. Here, we briefly outline the model and focus on the modifications. A more detailed description, including a parameter and resolution study, can be found in Rohde et al. (2019).

As in most subgrid outflow models we assume that the mass ejection rate is a fixed fraction of the accretion rate,

$$
\left.\frac{\mathrm{d} M}{\mathrm{~d} t}\right|_{\mathrm{EJECT}}=f_{\mathrm{EJECT}} \frac{\mathrm{d} M_{\star}}{\mathrm{d} t} .
$$

Here we adopt the default value $f_{\mathrm{EJECT}}=0.1$, based on observations and theoretical studies (see Croswell, Hartmann \& Avrett 1987; Shu et al. 1988; Pelletier \& Pudritz 1992; Calvet, Hartmann \& Kenyon 1993; Hartmann \& Calvet 1995; Nisini et al. 2018, or the review by Bally 2016). In contrast to most other subgrid outflow models, we do not use the accretion rate on to the sink particle, $\frac{\mathrm{d} M_{\mathrm{SINK}}}{\mathrm{d} t}$, but the episodic accretion rate on to the central star, $\frac{\mathrm{d} M_{\star}}{\mathrm{d} t}$ (Section 2.3). This leads to the intermittent ejection of individual outflow bullets (Rohde et al. 2019). To model the density and velocity distribution of the outflowing gas, we use the prescription for hydrodynamical 
outflows derived by Matzner \& McKee (1999). In this way we obtain a two-component outflow, with a collimated high-velocity jet, and a low-velocity wide-angle disc wind.

For the outflow velocity we assume

$v_{\mathrm{OUT}}=\left(\frac{\mathrm{G} M_{\star}}{r_{\mathrm{LAUNCH}}}\right)^{1 / 2} P(\theta)$,

which is the Keplerian velocity at radius $r_{\mathrm{LAUNCH}}$, modulated with the angular distribution, $P(\theta)$, derived by Matzner \& McKee (1999). Here, $\theta$ is the angle at which the SPH particle is ejected relative to the spin axis of the accretion disc. In contrast to Rohde et al. (2019) we do not adopt a fixed value for the launching radius, $r_{\text {LAUNCH }}$. Instead we use a time-dependent radius depending on the stellar radius, $R_{\star}$, provided by the stellar evolution model,

$r_{\mathrm{LAUNCH}}=2 R_{\star}$.

This gives us a more physically motivated outflow velocity, and avoids the need to invoke an arbitrary launching radius.

Outflows play a crucial role in removing angular momentum from the gas that is about to be accreted (Hartmann \& Stauffer 1989; Matt \& Pudritz 2005). Recent observations show that outflows are rotating and thus carry away angular momentum (Launhardt et al. 2009; Chen et al. 2016; Lee et al. 2017; Tabone et al. 2017). We incorporate rotating outflows by adding to the outward velocity, $\boldsymbol{v}_{\text {OUT }}$, a rotational velocity component,

$\boldsymbol{v}_{\mathrm{ROT}}=\boldsymbol{r} \times \boldsymbol{\omega}$

with

$\boldsymbol{\omega}=\frac{\ell_{\mathrm{SPH}}}{m_{\mathrm{SPH}} \sin ^{2}(\theta) r^{2}} \hat{\mathbf{e}}_{\mathrm{IAD}}$.

Here, $m_{\mathrm{SPH}}$ is the mass of an SPH particle and $\hat{\mathrm{e}}_{\mathrm{IAD}}=\boldsymbol{L}_{\mathrm{IAD}} /\left|\boldsymbol{L}_{\mathrm{IAD}}\right|$ is the spin axis of the IAD. In contrast to Rohde et al. (2019), we calculate the angular momentum each ejected particle carries away, $\ell_{\mathrm{PART}}$, from the breakup angular momentum of the protostar,

$L_{\text {BREAKUP }}=M_{\star} \sqrt{G M_{\star} R_{\star}} ;$

this assumes that the protostar rotates at its breakup angular speed. Whenever angular momentum is accreted from the IAD on to the central protostar, we compute the excess angular momentum,

$\ell_{\mathrm{SPH}}=\frac{\left|\boldsymbol{L}_{\star}\right|-L_{\mathrm{BREAKUP}}}{N_{\mathrm{EJECT}}}$,

allocate it to the ejected particles, and reduce $\left|\boldsymbol{L}_{\star}\right|$ to $L_{\text {BREAKUP }} ; N_{\text {EJECT }}$ is the number of ejected particles during this time-step.

\subsection{Simulation setup}

We have performed 88 simulations with different initial conditions or physical processes. All simulations start from a spherically symmetric, dense core with $M_{\mathrm{CORE}}=1 \mathrm{M}_{\odot}$ embedded in a lowdensity envelope at $T=10 \mathrm{~K}$. The density profile follows the radial distribution of a Bonnor-Ebert sphere (BES; Bonnor 1956; Ebert 1957). To obtain cores with $M_{\mathrm{CORE}}=1 \mathrm{M}_{\odot}$, we first construct a critical BES, truncated at the critical dimensionless radius $\xi_{0}=6.5$. The central densities are chosen in such a way, that the masses of the BESs are $\mathrm{M}_{\odot} / 3, \mathrm{M}_{\odot} / 4$, and $\mathrm{M}_{\odot} / 5$, corresponding to physical core radii of $r_{\mathrm{CORE}}=0.017,0.013$, and $0.010 \mathrm{pc}$, respectively. Then we increase the central densities by factors of $f_{\mathrm{BES}}=3,4$, or 5 , respectively, to $\rho_{\text {CENTRAL }}=2.0 \times 10^{-17}, 4.8 \times 10^{-17}$, or $9.4 \times 10^{-17} \mathrm{~g} \mathrm{~cm}^{-3}$ so that all the cores have $M_{\mathrm{CORE}}=1 \mathrm{M}_{\odot}$. This makes the cores more and more supercritical with increasing $f_{\mathrm{BES}}$. Thus, cores with higher
$f_{\mathrm{BES}}$ are smaller, denser and have shorter free-fall times, respectively, $t_{\mathrm{FF}}=36.8 \mathrm{kyr}, 24.6 \mathrm{kyr}$ and $16.6 \mathrm{kyr}$.

At $r_{\text {CORE }}$, the radial density profile decreases smoothly but quickly (power law with index $\gamma=-4$ ) to $\rho_{\mathrm{ENV}}=10^{-23} \mathrm{~g} \mathrm{~cm}^{-3}$. The envelope then extends to $r_{\mathrm{ENV}}=0.75 \mathrm{pc}$, which allows us to study the interaction of outflows with a low-density ambient medium. The total mass of the core plus envelope is $M_{\text {TOTAL }} \sim 1.86 \mathrm{M}_{\odot}$; this mass varies by at most 0.2 per cent due to varying $r_{\mathrm{CORE}}$.

As in Walch et al. (2010), we add an isotropic random Gaussian velocity field to the dense cores, in order to study the influence of turbulence on core collapse. The amplitudes follow a power spectrum of the form

$P_{k} \propto k^{-4}$ with $k \in\left[k_{\mathrm{MIN}}, 64\right]$.

Due to the steep power spectrum, most of the turbulent energy is associated with the smallest wavenumber, $k_{\text {MIN }}$. We stipulate $k_{\text {MIN }}=$ 1,2 or 3 , with $\frac{2 \pi}{k_{\text {miv }}}=1$ corresponding to the core diameter. In this way we change the velocity field from large-scale motions $\left(k_{\mathrm{MIN}}=1\right)$ with high net angular momentum, to small-scale turbulence $\left(k_{\mathrm{MIN}}=\right.$ 3 ) with low net angular momentum (Walch, Whitworth \& Girichidis 2012). We vary the strength of the turbulence by adjusting the virial ratio

$\alpha_{\mathrm{VIR}}=\frac{2\left(E_{\mathrm{TURB}}+E_{\mathrm{THERM}}\right)}{\left|E_{\mathrm{GRAV}}\right|}$.

We perform simulations for all combinations of $\alpha_{\mathrm{VIR}}=0.5,1.0,2.0$, and 3.0, $\quad k_{\mathrm{MIN}}=1,2$, and 3, and $r_{\mathrm{CORE}}=0.010,0.013$, and $0.017 \mathrm{pc}$. In addition, we perform runs with $\alpha_{\mathrm{VIR}}=1.0, k_{\mathrm{MIN}}=1$, and $r_{\mathrm{CORE}}=0.013 \mathrm{pc}$ for eight different turbulent seeds. To study the influence of outflow feedback on the SFE we produce a comparison run without outflow feedback for each setup. This adds up to 88 simulations in total (see Table 1). The mass resolution is $400000 \mathrm{SPH}$ particles per $\mathrm{M}_{\odot}$, resulting in a total number of $N_{\text {TOTAL }} \sim 740000 \mathrm{SPH}$ particles.

\section{RESULTS}

Due to their different initial conditions, some simulations form stars faster than others. To carry out objective comparisons between the simulations, we make them at times $t_{\tau}$ where $t_{\tau}=t_{0}+\tau t_{\mathrm{FF}}$; here $t_{0}$ is the time at which the first sink forms, $t_{\mathrm{FF}}=\pi\left(r_{\mathrm{CORE}}^{3} /\left(8 G M_{\mathrm{CORE}}\right)^{1 / 2}\right.$ is the core's free-fall time, and we use $\tau=0.5,1.5$, and 5.0. All simulations are terminated at $t_{5} \sim 200 \pm 50 \mathrm{kyr}$. The ensemble of simulations is divided into those with outflow feedback (the OFsample, with odd IDs and run names ending in 'O-1') and those without outflow feedback (RF-sample, with even IDs and run names ending in ' $\mathrm{O}-0$ '). Both samples contain 44 simulations (Table 1). The OF-sample forms $N_{\star \mathrm{O}-1}=132$ stars in total, whereas the RF-sample forms $N_{\star 0-0}=163$. All statistical tests use a significance threshold of $p<1$ per cent.

\subsection{Stellar diversity}

The ensemble of simulations produces a wide variety of stellar configurations: single stars and multiple systems; circumstellar and/or circumbinary discs; aligned and misaligned outflows. Fig. 1 illustrates four representative runs with outflow feedback, all at $t_{0.5}$. The green markers represent sink particles. The left-hand column shows the central regions around the sink particles and their accretion discs. The right-hand column shows the outflows on larger scales.

The simulation on the top row of Fig. 1 (S-5_V-2.0_K-3_R0.017_O-1) forms a wide binary system with a circumbinary 
Table 1. Parameter summary for all the simulations performed. Reading from left to right the columns give the run number, the run name, the turbulent random seed $(\chi)$, the virial ratio $\left(\alpha_{\mathrm{VIR}}\right)$, the smallest turbulent wavenumber $\left(k_{\mathrm{MIN}}\right)$, and the core radius $\left(r_{\mathrm{CORE}} / \mathrm{pc}\right)$. Each combination of parameters is simulated once with, and once without, outflow feedback. The simulations with outflow feedback have odd IDs and their run names end with $x=1$. The simulations without outflow feedback have even IDs and their run names end with $x=0$.

\begin{tabular}{|c|c|c|c|c|c|}
\hline \# & Run & $\chi$ & $\alpha_{\mathrm{VIR}}$ & $k_{\mathrm{MIN}}$ & $r_{\mathrm{CORE}}$ \\
\hline $1 / 2$ & S-1_V-0.5_K-1_R-0.017_O- $x$ & 5 & 0.5 & 1 & 0.017 \\
\hline $3 / 4$ & S-1_V-1.0_K-1_R-0.017_O-x & 5 & 1.0 & 1 & 0.017 \\
\hline $5 / 6$ & S-1_V-2.0_K-1_R-0.017_O- $x$ & 5 & 2.0 & 1 & 0.017 \\
\hline $7 / 8$ & S-1_V-3.0_K-1_R-0.017_O- $x$ & 5 & 3.0 & 1 & 0.017 \\
\hline $9 / 10$ & S-1_V-0.5_K-2_R-0.017_O- $x$ & 5 & 0.5 & 2 & 0.017 \\
\hline $11 / 12$ & S-1_V-1.0_K-2_R-0.017_O- $x$ & 5 & 1.0 & 2 & 0.017 \\
\hline $13 / 14$ & S-1_V-2.0_K-2_R-0.017_O- $x$ & 5 & 2.0 & 2 & 0.017 \\
\hline $15 / 16$ & S-1_V-3.0_K-2_R-0.017_O-x & 5 & 3.0 & 2 & 0.017 \\
\hline $17 / 18$ & S-1_V-0.5_K-3_R-0.017_O- $x$ & 5 & 0.5 & 3 & 0.017 \\
\hline $19 / 20$ & S-1_V-1.0_K-3_R-0.017_O- $x$ & 5 & 1.0 & 3 & 0.017 \\
\hline $21 / 22$ & S-1_V-2.0_K-3_R-0.017_O- $x$ & 5 & 2.0 & 3 & 0.017 \\
\hline $23 / 24$ & S-1_V-3.0_K-3_R-0.017_O- $x$ & 5 & 3.0 & 3 & 0.017 \\
\hline $25 / 26$ & S-1_V-0.5_K-1_R-0.013_O-x & 5 & 0.5 & 1 & 0.013 \\
\hline $27 / 28$ & S-1_V-1.0_K-1_R-0.013_O-x & 5 & 1.0 & 1 & 0.013 \\
\hline $29 / 30$ & S-1_V-2.0_K-1_R-0.013_O-x & 5 & 2.0 & 1 & 0.013 \\
\hline $31 / 32$ & S-1_V-3.0_K-1_R-0.013_O- $x$ & 5 & 3.0 & 1 & 0.013 \\
\hline $33 / 34$ & S-1_V-0.5_K-2_R-0.013_O- $x$ & 5 & 0.5 & 2 & 0.013 \\
\hline $35 / 36$ & S-1_V-1.0_K-2_R-0.013_O- $x$ & 5 & 1.0 & 2 & 0.013 \\
\hline $37 / 38$ & S-1_V-2.0_K-2_R-0.013_O- $x$ & 5 & 2.0 & 2 & 0.013 \\
\hline $39 / 40$ & S-1_V-3.0_K-2_R-0.013_O- $x$ & 5 & 3.0 & 2 & 0.013 \\
\hline $41 / 42$ & S-1_V-0.5_K-3_R-0.013_O-x & 5 & 0.5 & 3 & 0.013 \\
\hline $43 / 44$ & S-1_V-1.0_K-3_R-0.013_O- $x$ & 5 & 1.0 & 3 & 0.013 \\
\hline $45 / 46$ & S-1_V-2.0_K-3_R-0.013_O- $x$ & 5 & 2.0 & 3 & 0.013 \\
\hline $47 / 48$ & S-1_V-3.0_K-3_R-0.013_O- $x$ & 5 & 3.0 & 3 & 0.013 \\
\hline $49 / 50$ & S-1_V-0.5_K-1_R-0.010_O- $x$ & 5 & 0.5 & 1 & 0.010 \\
\hline $51 / 52$ & S-1_V-1.0_K-1_R-0.010_O- $x$ & 5 & 1.0 & 1 & 0.010 \\
\hline $53 / 54$ & S-1_V-2.0_K-1_R-0.010_O- $x$ & 5 & 2.0 & 1 & 0.010 \\
\hline $55 / 56$ & S-1_V-3.0_K-1_R-0.010_O- $x$ & 5 & 3.0 & 1 & 0.010 \\
\hline $57 / 58$ & S-1_V-0.5_K-2_R-0.010_O- $x$ & 5 & 0.5 & 2 & 0.010 \\
\hline $59 / 60$ & S-1_V-1.0_K-2_R-0.010_O- $x$ & 5 & 1.0 & 2 & 0.010 \\
\hline $61 / 62$ & S-1_V-2.0_K-2_R-0.010_O- $x$ & 5 & 2.0 & 2 & 0.010 \\
\hline $63 / 64$ & S-1_V-3.0_K-2_R-0.010_O- $x$ & 5 & 3.0 & 2 & 0.010 \\
\hline $65 / 66$ & S-1_V-0.5_K-3_R-0.010_O- $x$ & 5 & 0.5 & 3 & 0.010 \\
\hline $67 / 68$ & S-1_V-1.0_K-3_R-0.010_O- $x$ & 5 & 1.0 & 3 & 0.010 \\
\hline $69 / 70$ & S-1_V-2.0_K-3_R-0.010_O- $x$ & 5 & 2.0 & 3 & 0.010 \\
\hline $71 / 72$ & S-1_V-3.0_K-3_R-0.010_O- $x$ & 5 & 3.0 & 3 & 0.010 \\
\hline $73 / 74$ & S-2_V-1.0_K-1_R-0.013_O- $x$ & 0 & 1.0 & 1 & 0.013 \\
\hline $75 / 76$ & S-3_V-1.0_K-1_R-0.013_O- $x$ & 1 & 1.0 & 1 & 0.013 \\
\hline $77 / 78$ & S-4_V-1.0_K-1_R-0.013_O-x & 2 & 1.0 & 1 & 0.013 \\
\hline $79 / 80$ & S-5_V-1.0_K-1_R-0.013_O- $x$ & 3 & 1.0 & 1 & 0.013 \\
\hline $81 / 82$ & S-6_V-1.0_K-1_R-0.013_O-X & 4 & 1.0 & 1 & 0.013 \\
\hline $83 / 84$ & S-7_V-1.0_K-1_R-0.013_O-x & 6 & 1.0 & 1 & 0.013 \\
\hline $85 / 86$ & S-8_V-1.0_K-1_R-0.013_O- $x$ & 7 & 1.0 & 1 & 0.013 \\
\hline $87 / 88$ & S-9_V-1.0_K-1_R-0.013_O- $x$ & 8 & 1.0 & 1 & 0.013 \\
\hline
\end{tabular}

disc. A third star forms in the circumbinary disc via discfragmentation. The binary system becomes an hierarchical triple system when the third star spirals inwards. The outflows from all three stars are well aligned and produce a broad outflow cavity.

The simulation on the second row of Fig. 1 (S-5_V-0.5_K-1_R0.010_O-1) also forms three stars that end up in a stable triple system. Two of these stars belong to a close binary system with a circumbinary disc, while the third star has its own circumstellar disc. These two systems are surrounded by a larger accretion disc, and material from this larger disc streams inwards along a spiral structure and on to the two smaller discs.

The simulation on the third row of Fig. 1 (S-5_V-3.0_K-1_R0.013_O-1) forms four stars in total. Initially these stars are in an hierarchical quadruple system (a close binary, a third star orbiting further out, and a fourth star orbiting even further out). This fourth star has the largest accretion disc, and there are spiral accretion flows feeding material inwards from larger scales and on to the accretion discs. Later on only the close binary remains bound.

The simulation on the bottom row of Fig. 1 (S-5_V-1.0_K-3_R0.017_O-1) forms two stars in a binary system. Both stars have their own circumstellar accretion discs, with a bridge in between. At this stage the outflows from the stars point in slightly different directions, but later on they align.

\subsection{Overview of stellar masses and multiplicities}

Fig. 2 shows, as a function of the total mass in stars, $M_{\star \text { тотАL }}$ at $t_{5}$, the number of stars formed in a core, $N_{\star}$ (top row), the mass of the most massive star, $M_{\star \mathrm{MM}}$ (second row), the order of the highest order system, $O_{\text {SYS-MAX }}$ (third row), and the total stellar mass of this highestorder system, $M_{\star \text { SYS-MAX }}$ (bottom row). The left-hand column shows the results for the OF-sample, and the right-hand column shows them for the RF-sample. The SFE is SFE $=M_{\star \text { TOTAL }} / M_{\mathrm{CORE}}$ at $t_{5}$. Since the core can accrete matter from its surroundings and convert this matter into stars, SFE can exceed unity.

The top row of Fig. 2 indicates that there is no significant correlation between $N_{\star}$ and $M_{\star \text { TотАL }}$, for either sample. A Kendall Rank Correlation (KRC) test confirms this, with $p \sim 30$ per cent for both samples. On average, the RF-sample forms more stars per core, $\bar{N}_{\star}(\mathrm{O}-0)=3.88 \pm 2.12$ than the OF-sample, $\bar{N}_{\star}(\mathrm{O}-1)=$ $3.14 \pm 1.95$. The theoretical model of Holman et al. (2013) predicts a slightly higher number, $N_{\star}=4.1 \pm 0.4$. The core that forms the highest number of stars, $N_{\star}=9$, is S-5_V-1.0_K-3_R-0.010_O-0 in the RF-sample. The OF-sample contains 13 simulations which form only a single star, as compared with only 5 in the RF-sample.

The second row of Fig. 2 shows that the ratio of the mass of the most massive star to the total stellar mass, $M_{\star \mathrm{MM}} / M_{\star \mathrm{TOTAL}}$, is between $\sim 0.2$ and $\sim 0.6$. For the OF-sample, this ratio shows no correlation with the total stellar mass, $M_{\star \text { ТотAL }}$. For the RF-sample, the ratio shows a slight tendency to increase with increasing $M_{\star \text { ॠотAL }}$, but with a large scatter.

The third row of Fig. 2 shows that the order of the highest-order system formed in each core, $O_{\text {SYS-MAX }}$, is not significantly correlated with $M_{\star \mathrm{TOTAL}} \cdot{ }^{1}$ Multiple systems are identified and characterized using the method proposed by Lomax et al. (2015), which iteratively pairs up stars and multiples in an hierarchical order, taking into account their mutual gravitational and kinetic energies, their eccentricity, and whether the pair is tidally bound. Many single stars are ejected by dynamical interactions with multiples. We discuss these ejected stars in Section 4.

Strictly speaking, only binary systems are truly stable, in the sense that they can survive indefinitely, in isolation. However, HOMs can survive for a very long time if they are arranged hierarchically. Consequently, some of them will survive long after the dispersal of the birth core, but many will end up as binaries, and some will dissolve completely into singles (e.g. run S-5_V-1.0_K-3_R-0.010_O1). In general, the larger the number of stars, the larger the number of

${ }^{1}$ The highest-order system formed in a core is not necessarily a higher-order multiple (HOM), it could be a single or a binary. 

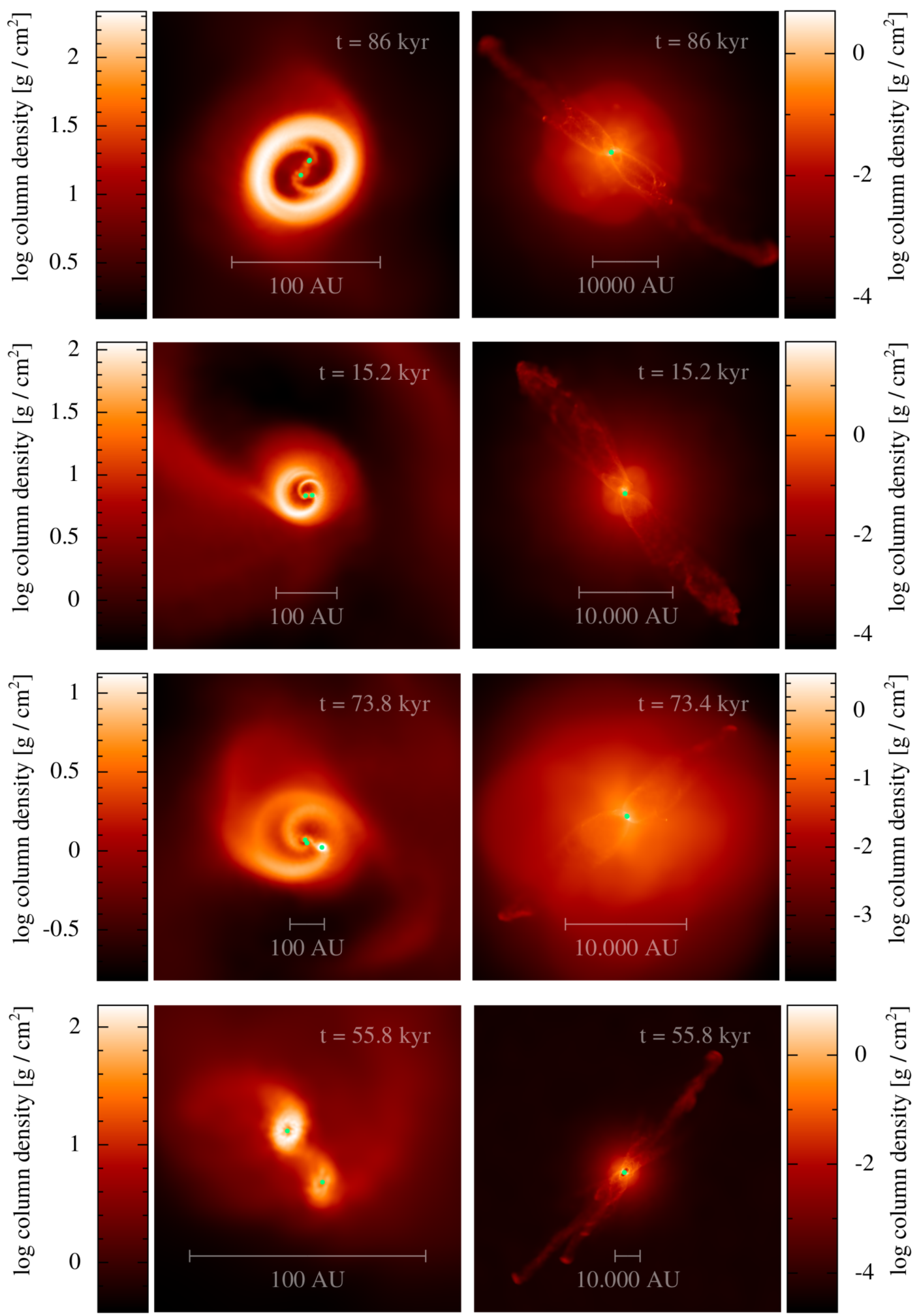

Figure 1. Column density plots of four representative simulations with outflow feedback at $t_{0.5} \equiv t_{0}+0.5 t_{\mathrm{FF}}$. The left-hand column shows the multiple systems and accretion discs in the central regions. The right-hand column shows the same simulations, but zoomed out to reveal their outflows. The green dots represent sink particles. The simulations are from top to bottom S-5_V-2.0_K-3_R-0.017_O-1, S-5_V-0.5_K-1_R-0.010_O-1, S-5_V-3.0_K-1_R-0.013_O-1, and S-5_V-1.0_K-3_R-0.017_O-1. Note that the scale, the colour bar, and the viewing-angle are different for each panel.

ejected singles. For example, run S-5_V-1.0_K-3_R-0.010_O-0 forms nine stars, but ejects six of them and ends up as an hierarchical triple system. In all simulations that form only two stars, these two always end up in a binary.
One very striking difference between the OF- and RF-samples is the fractions of single $\left(S_{1}\right)$, binary $\left(B_{2}\right)$, triple $\left(T_{3}\right)$, and quadruple $\left(Q_{4}\right)$ systems formed. For the OF-sample, there is a monotonic decrease with increasing order, viz. $\left(S_{1}: B_{2}: T_{3}: Q_{4}\right)=(0.38: 0.29: 0.24: 0.10)$. 

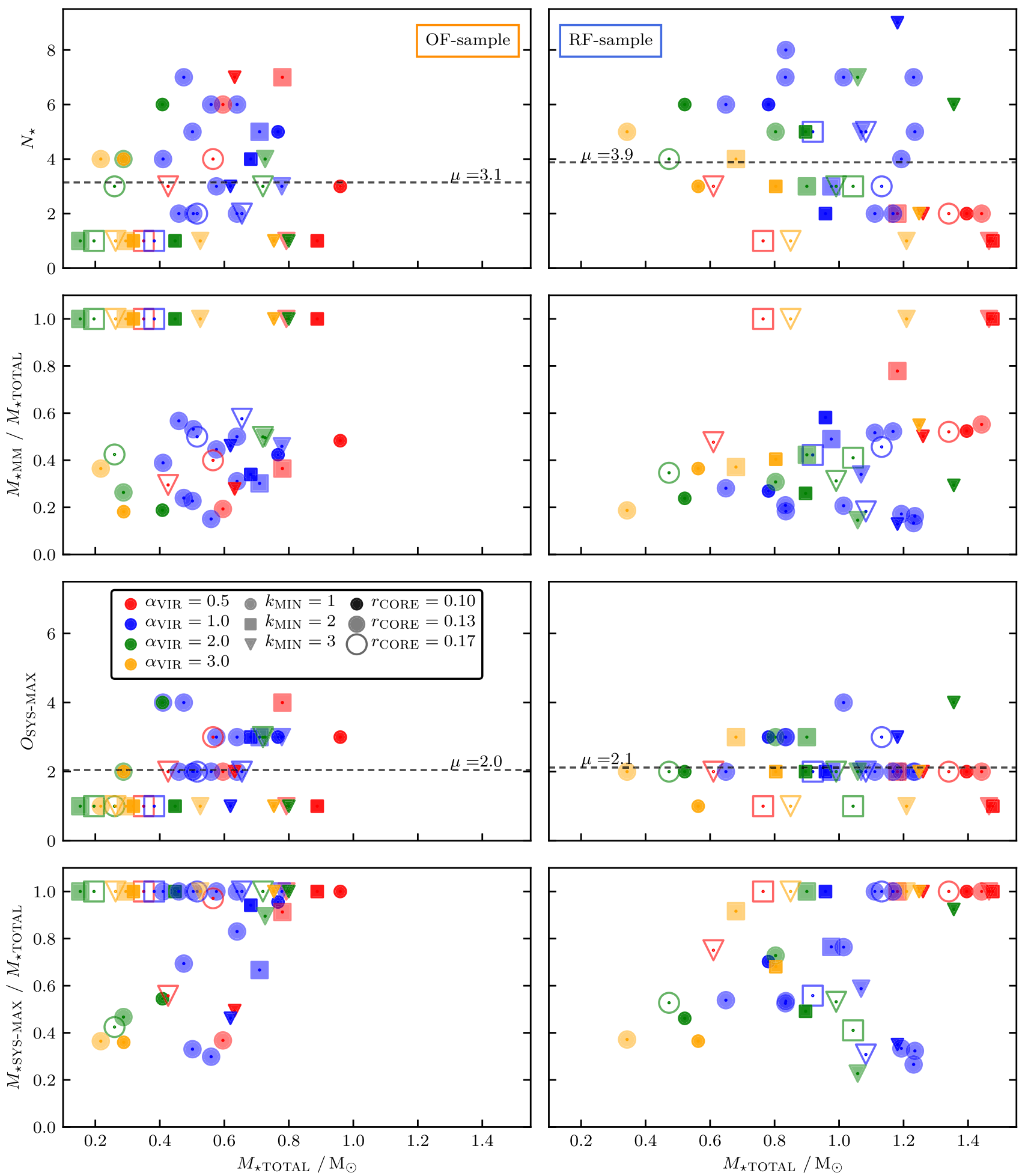

Figure 2. Scatter plots showing the properties of the stars formed in a core against total mass of stars, for all the simulations, at $t_{5} \equiv t_{0}+5 t_{\mathrm{FF}}$. The left-hand column shows the OF sample, and the right-hand column shows the RF-sample. The size of the symbol encodes the core's initial radius, $r_{\mathrm{CORE}}$; the colour of the symbol encodes the core's initial virial parameter, $\alpha_{\mathrm{VIR}}$; and the shape of the symbol encodes the wavenumber of the largest initial turbulent mode in the core (see key on third panel down, left-hand side). The top row shows the total number of stars, $N_{\star}$, and the dashed lines indicate the mean values, $\bar{N}_{\star}(O-1)=3.14 \pm 1.95$ and $\bar{N}_{\star}(O-0)=3.88 \pm 2.12$. The second row shows the mass of the most massive star, as a fraction of the total mass of stars, $M_{\star \mathrm{MM}} / M_{\star \text { TOTAL}}$. The third row shows the order of the highest-order system, and the dashed lines indicate the mean values, $\bar{O}_{\mathrm{SYS}}(O-1)=2.05 \pm 1.00$ and $\bar{O}_{\text {SYS }}(O-0)=2.12 \pm 0.73$. The bottom row shows the mass of the highest-order system, as a fraction of the total mass of stars, $M_{\star \text { sYS-MAX }} / M_{\star \text { TOTAL }}$. 


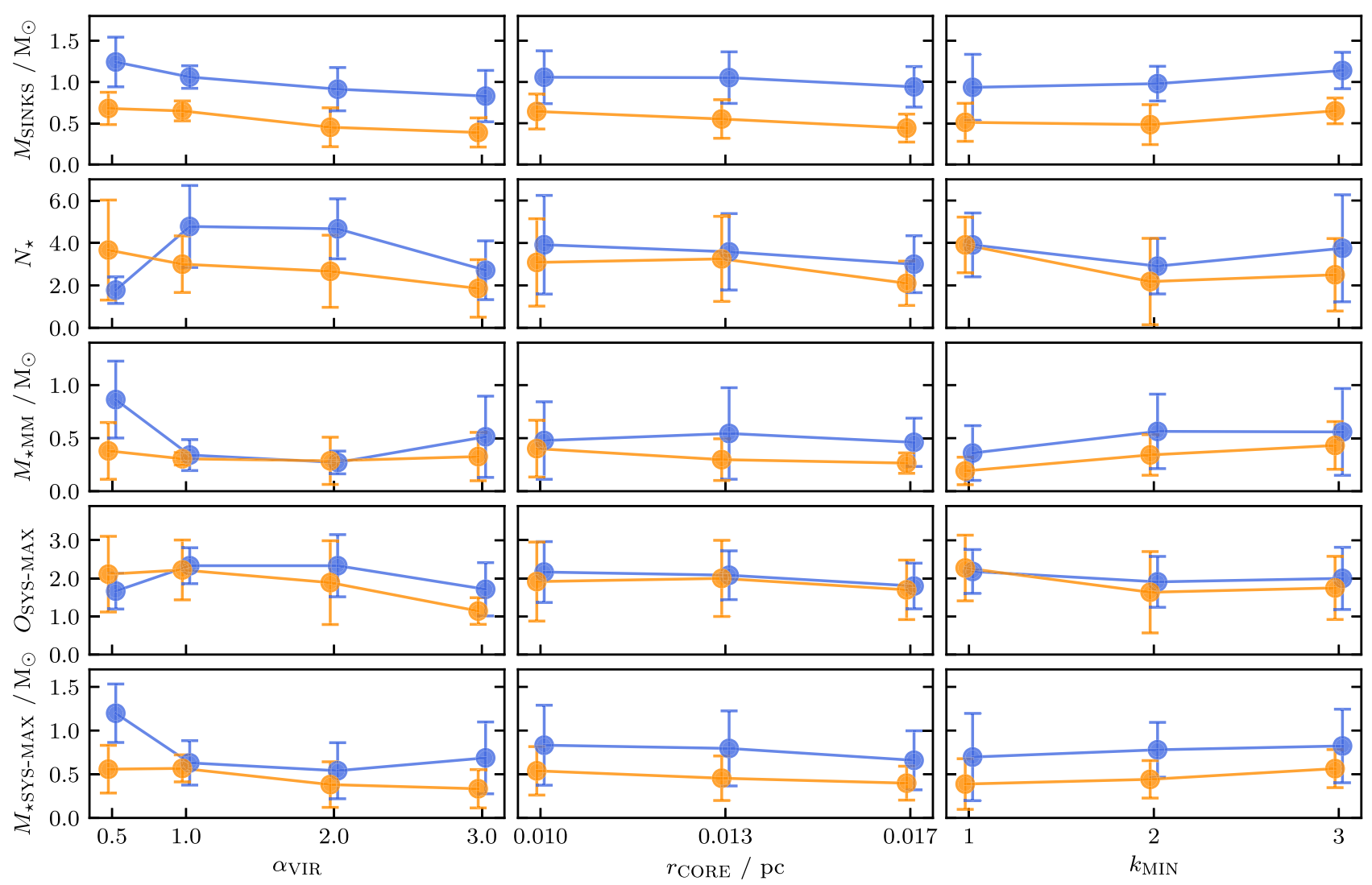

Figure 3. Mean values for the stellar parameters plotted in Fig. 2, as a function of the parameters defining the initial conditions of the birth core. Simulations from the OF-sample are plotted in orange, and those from the RF-sample are plotted in blue. The left-hand column shows the means for subsets with the same $\alpha_{\mathrm{VIR}}=0.5,1.0,2.0$ or 3.0 (i.e. averaged over all values of $r_{\mathrm{CORE}}$ and $k_{\mathrm{MIN}}$ ). The middle column shows the means for subsets with the same $r_{\mathrm{CORE}}=0.010 \mathrm{pc}, 0.013 \mathrm{pc}$ or $0.017 \mathrm{pc}$ (i.e. averaged over all values of $\alpha_{\mathrm{VIR}}$ and $k_{\mathrm{MIN}}$ ). The right-hand column shows the means for subsets with the same $k_{\mathrm{MIN}}=1,2$ or 3 (i.e. averaged over all values of $\alpha_{\mathrm{VIR}}$ and $r_{\mathrm{CORE}}$ ). The top row shows the mean total stellar mass, $\bar{M}_{\star \text { TOTAL }}$; the second row shows the mean number of stars, $\bar{N}_{\star}$; the third row shows the mean mass of the most massive star, $\bar{M}_{\star \mathrm{MM}}$; the fourth and fifth rows show the mean order, $\bar{O}_{\text {SYs }}$, and the mean mass, $\bar{M}_{\star \text { SYS-MAX }}$ of the highest-order system. The initial conditions of the birth core appear to have very limited influence on the properties of the stars formed.

In contrast, the RF-sample mainly forms binary systems, $\left(S_{1}: B_{2}: T_{3}\right.$ : $\left.Q_{4}\right)=(0.17: 0.60: 0.19: 0.05)$. The fraction of triple and quadruple systems is slightly higher for the OF-sample. However, due to the high fraction of binaries in the RF-sample, the mean orders of the largest systems are very similar: $O_{\text {SYS-MAX }}=2.0$ for the OF-sample, and $O_{\text {SYS-MAX }}=2.1$ for the RF-sample.

The bottom row of Fig. 2 shows the ratio of the mass in the highestorder system to the total stellar mass, $M_{\star \text { SYS-MAX }} / M_{\star \text { TOTAL }}$. The OFsample has significantly more simulations with $M_{\star S \mathrm{YS}-\mathrm{MAX}} / M_{\star \mathrm{TOTAL}}=$ 1.0, because many more simulations form just a single star. Setting aside the systems with a ratio close to one, $M_{\star \text { SYS-MAX }} / M_{\star \text { TOTAL }}$ tends to increase with increasing $M_{\star \text { TOTAL }}$, up to $M_{\star \text { TOTAL }} \sim 0.8 \mathrm{M}_{\odot}$, for both samples, albeit with large scatter. Above $M_{\star \text { TOTAL }} \sim 0.8 \mathrm{M}_{\odot}$, there are no multiple systems in the OF-sample, but for the RF-sample $M_{\star \mathrm{SYS}-\mathrm{MAX}} / M_{\star \mathrm{TOTAL}}$ then tends to decrease with increasing $M_{\star \text { TOTAL }}$; this is because these simulations produce large numbers of stars and only a few of them end up in the highest-order system.

\subsection{Influence of initial core properties}

The initial conditions for the simulated cores are characterized by four parameters: the core radius, which takes values $r_{\mathrm{CORE}}=$ $0.010 \mathrm{pc}, 0.013 \mathrm{pc}$ and $0.017 \mathrm{pc}$; the virial ratio, which takes values $\alpha_{\mathrm{VIR}}=0.5,1.0,2.0$ and 3.0; the minimum wavenumber for the imposed turbulent modes, which takes values $k_{\mathrm{MIN}}=1,2$ and 3; and the seed for the random turbulent modes excited, which takes the same value $\chi=1$ for all combinations of $\left(r_{\text {CORE }}, \alpha_{\mathrm{VIR}}, k_{\mathrm{MIN}}\right)$ except for $\left(r_{\mathrm{CORE}}, \alpha_{\mathrm{VIR}}, k_{\mathrm{MIN}}\right)=(0.013 \mathrm{pc}, 1.0,1)$, for which we perform runs with $\chi=1,2,3,4,5,6,7$, and 8 .

In order to explore how these parameters influence the masses and multiplicities of the stars formed in a core, we compute average values at $t_{5} \equiv t_{0}+5 t_{\mathrm{FF}}$ for (i) the total stellar mass, $M_{\star \text { TотAL }}$, (ii) the number of stars, $N_{\star}$, (iii) the mass of the most massive star, $M_{\star \mathrm{MM}}$, (iv) the order of the highest-order system, $O_{\mathrm{SYS}}$, and (v) the mass of the highest order system, $M_{\star \text { SYS-MAX }}$, for all the simulations with a given radius $r_{\mathrm{CORE}}$ but different values of $\alpha_{\mathrm{VIR}}$ and $k_{\mathrm{MIN}}-$ and similarly for all the simulations with a given virial parameter $\alpha_{\mathrm{VIR}}$ but different $r_{\mathrm{CORE}}$ and $k_{\mathrm{MIN}}$, and all the simulations with a given minimum turbulent wavenumber $k_{\mathrm{MIN}}$ but different $r_{\mathrm{CORE}}$ and $\alpha_{\mathrm{VIR}}$. The results are presented on Fig. 3, where the results for simulations from the OF-sample are in orange, and those from the RF-sample are in blue.

To quantify the results presented in Fig. 3 we evaluate the dependence of these mean stellar parameters $\left(\bar{M}_{\star \mathrm{TOTAL}}, \bar{N}_{\star}, \bar{M}_{\star \mathrm{MM}}, \bar{O}_{\mathrm{SYS}}, \bar{M}_{\star \mathrm{SYS}-\mathrm{MAX}}\right)$ on the initial condition parameters $\left(\alpha_{\mathrm{VIR}}, r_{\mathrm{CORE}}, k_{\mathrm{MIN}}\right)$ by computing the Kendall Rank Correlation statistics, $\tau$ and $p$ (see Table 2); $\tau$ gives the degree of correlation (or anticorrelation, if negative). In addition, we evaluate whether the OF- and RF-samples are drawn from the same underlying distribution, by computing the non-parametric Kolmogorov-Smirnov 
Table 2. Non-parametric measures of the correlations, and their statistical significances, for the data presented in Fig. 3. The left double-column gives the stellar parameters considered, in the same order as in Fig. 3, and the sample used (outflow OF or reference RF). The second double-column gives the Kolmogorov-Smirnov statistics, $d$ and $p$, which reflect the likelihood that the two samples are drawn from the same distribution. The last three double-columns give the Kendall Rank Correlation (KRC) statistics, $\tau$ and $p$, which reflect the likelihood that the stellar parameters are correlated with, respectively, $\alpha_{\mathrm{VIR}}, r_{\mathrm{CORE}}$, and $k_{\mathrm{MIN}}$; the KRC statistics are evaluated separately for the OF- and RF-samples. Correlations that satisfy our significance threshold of $p<1 \%$ are highlighted.

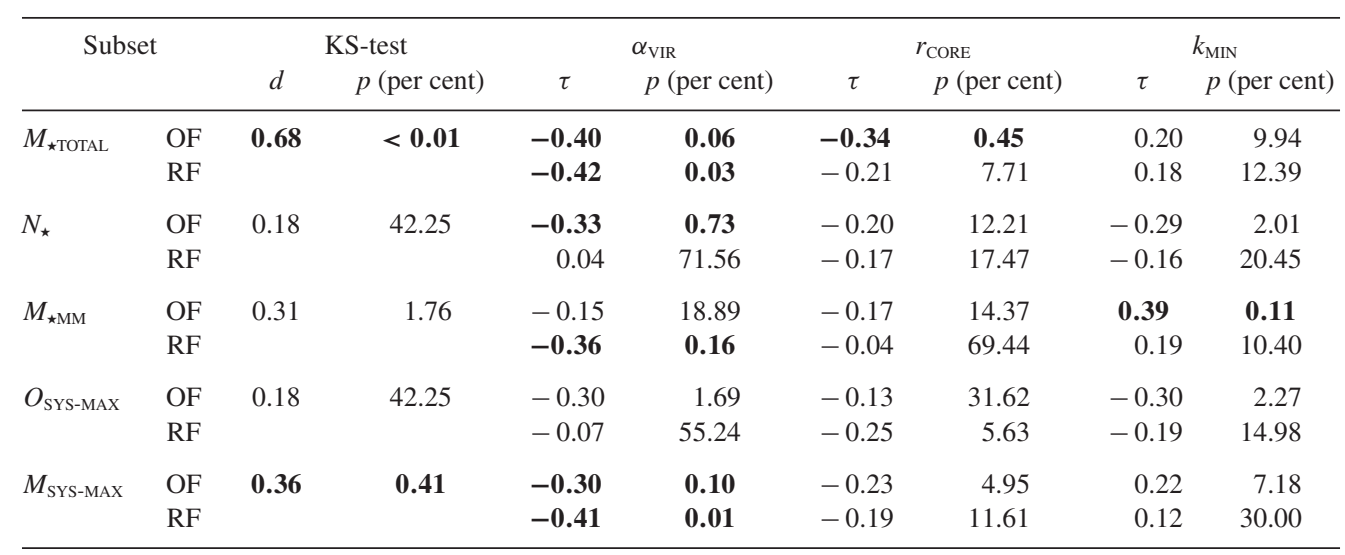

(KS) statistics, $d$ and $p ; d$ measures the difference between the two distributions. In both cases, $p$ gives the probability of obtaining the evaluated correlation $(\tau)$ or difference $(d)$ assuming the null hypothesis (i.e. that both samples are drawn from the same underlying distribution).

Fig. 3 and Table 2 demonstrate clearly that varying the initial conditions - at least in the range we have studied - has little influence on the properties of the stars formed. The correlations between stellar parameters and initial condition parameters are at best weak $(|\tau| \leq$ 40), and in most cases they are not significant, so we only discuss those for which $p<1$ per cent. For both samples (OF and RF), $\bar{M}_{\star \text { TOTAL }}$ decreases with increasing $\alpha_{\mathrm{VIR}}$ (because the cores have more support and collapse more slowly); for the OF-sample, $\bar{M}_{\star \text { TOTAL }}$ also decreases with increasing $r_{\text {CORE }}$ (first because the cores collapse more slowly, and secondly because the outflow feedback acts on more rarefied gas and is therefore more effective). For the OF-sample, $\bar{N}_{\star}$ decreases with increasing $\alpha_{\text {VIR }}$ (because the cores have more support and are therefore more easily dispersed by outflow feedback). $\bar{M}_{\star \mathrm{MM}}$ increases with increasing $k_{\mathrm{MIN}}$ for the OF-sample (because the turbulence is concentrated on small scales which dissipate more rapidly), and with decreasing $\alpha_{\mathrm{VIR}}$ for the RF-sample (because the cores have less turbulent support and therefore their collapse is more focused). $\bar{M}_{\star \text { SYS-MAX }}$ increases with decreasing $\alpha_{\text {VIR }}$ for both samples (again, because the cores have less turbulent support and therefore their collapse is more focused).

The one exception to these correlations, anticorrelations and insignificant correlations is the $\alpha_{\mathrm{VIR}}=0.5 \mathrm{RF}$ subset, which bucks most of the trends seen in the other subsets. The very low level of core support $\left(\alpha_{\mathrm{VIR}}=0.5\right)$ and the lack of outflow feedback (RF) result in a rather focused infall on to the centre of the core, and consequently the formation of either a massive single star, or a massive binary (usually with approximately equal-mass components).

The second double column of Table 2 demonstrates that the OFand RF-samples are statistically distinct. In particular, $\bar{M}_{\star \text { TOTAL }}$ is almost twice as large for the RF-sample as for the OF-sample (see Fig. 3, top panel). $\bar{M}_{\star \mathrm{MM}}$ and $\bar{M}_{\star \text { SYS-MAX }}$ are also larger for the RFsample than the OF-sample, by $\sim 50$ per cent (see Fig. 3, third and bottom panels). These differences are mainly due to the fact that in the OF simulations the outflow feedback disperses the gas surrounding the core, but in the RF simulations the surrounding gas falls on to the core and replenishes its mass. $\bar{N}_{\star}$ and $\bar{O}_{\text {SYS }}$ are indistinguishable between the two samples.

\subsection{Influence of turbulent seeds}

To make sure our results are not dominated by the particular choice of the turbulent velocity field for the fiducial runs, we perform eight additional runs with different random turbulent seeds (Table 1, runs with number 73-88), with and without outflow feedback. These runs have otherwise the same initial condition values as our fiducial runs S-5_V-1_K-1_R-4_O-x. In Fig. 2 the subset of these runs $(\chi-$ subset) are represented by the middle-sized, blue shaded circles. The spread of the ( $\chi$-subset) is comparable to the spread of the runs with varying initial conditions (IC-subset). Remarkable is that the $\chi$-subset contains no run that forms only a single star.

Table 3 gives the mean and standard deviation of the full sample (OF and RF), the $\chi$-subset and the IC-subset for all quantities presented in Figs 2 and 3. The mean values and their spread are comparable for both samples. Two differences, however not statistically significant, are that the $\chi$-subset (a) forms on average slightly more stars and (b) has slightly lower masses of the most massive star due to the absence of runs forming a single star. The last two columns of Table 3 give the Kolmogorov-Smirnov statistics, $d$ and $p$, reflecting the difference between the $\chi$ - and ICsubset and the probability of finding these results assuming the null hypothesis is true. We do not find a statistical difference between the two subsets, and we are unable to reject the null hypothesis that both subsets have the same underlying distribution. However, we caution that this is not a proof that the distributions are the same.

The similarity between the $\chi$-subset and the IC-subset makes us confident that our results are not dominated by the choice of the random seeds. On the other hand, this finding supports our result from Section 3.3. Since the influence of the varying initial conditions on the outcome of the simulation is not higher than the influence due to different turbulent seeds, the core properties play at most a limited role in the outcome of the simulation. 
Table 3. Mean and standard deviation for the quantities presented in Fig. 2 for the subsets with varying turbulent seeds and initial conditions (IC). The first column gives the quantities presented in Fig. 2, the second column the feedback mechanism. The third, fourth, and fifth column give the mean and standard deviation for the full OF- and RF-samples (full-sample), the reduced subset with varying turbulent seeds ( $\chi$-subset) and the reduced subset with varying initial condition parameters (IC-subset). The sixth and seventh column give the Kolmogorov-Smirnov statistics, $d$ and $p$, which reflect the likelihood that the $\chi$ - and IC-sample are drawn from the same distribution.

\begin{tabular}{lcccccc}
\hline Quantity & Feedback & Full-sample & $\chi$-subset & IC-subset & $d$ & $p$ (percent) \\
\hline $\bar{M}_{\star \text { TOTAL }}$ & OF & $0.54 \pm 0.20$ & $0.53 \pm 0.08$ & $0.54 \pm 0.22$ & 0.38 & 19.04 \\
& RF & $1.00 \pm 0.28$ & $1.03 \pm 0.20$ & $1.00 \pm 0.30$ & 0.24 & 74.01 \\
$\bar{N}_{\star}$ & OF & $3.14 \pm 1.95$ & $4.11 \pm 1.85$ & $2.85 \pm 1.87$ & 0.38 & 19.04 \\
& RF & $3.88 \pm 2.12$ & $5.33 \pm 2.11$ & $3.53 \pm 1.93$ & 0.41 & 14.00 \\
$\bar{M}_{\star \text { MM }}$ & OF & $0.30 \pm 0.20$ & $0.20 \pm 0.08$ & $0.32 \pm 0.21$ & 0.45 & 8.22 \\
& RF & $0.46 \pm 0.34$ & $0.27 \pm 0.17$ & $0.50 \pm 0.36$ & 0.54 & 2.82 \\
$\bar{O}_{\text {SYS-MAX }}$ & OF & $2.05 \pm 1.00$ & $2.67 \pm 0.82$ & $1.88 \pm 0.96$ & 0.47 & 6.81 \\
& RF & $2.12 \pm 0.73$ & $2.44 \pm 0.68$ & $2.03 \pm 0.71$ & 0.21 & 86.28 \\
$\bar{M}_{\text {SYS-MAX }}$ & OF & $0.45 \pm 0.24$ & $0.42 \pm 0.16$ & $0.47 \pm 0.25$ & 0.35 & 27.61 \\
& RF & $0.74 \pm 0.41$ & $0.60 \pm 0.31$ & $0.77 \pm 0.42$ & 0.37 & 21.49 \\
\hline
\end{tabular}

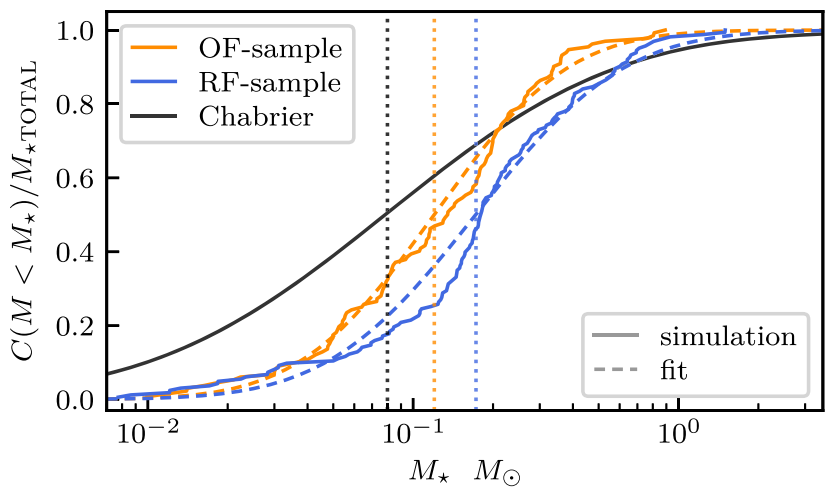

Figure 4. Cumulative mass functions for the OF-sample (132 stars, solid orange line) and the RF-sample (163 stars, solid blue line) at $t_{5}=t_{0}+5 t_{\mathrm{FF}}$. The dashed lines show lognormal fits to these distributions, and the dotted lines indicate the mean values, $\bar{M}_{\mathrm{OF}}=0.13 \mathrm{M}_{\odot}$ and $\bar{M}_{\mathrm{RF}}=0.18 \mathrm{M}_{\odot}$. The black line shows the Chabrier IMF for comparison $\left(\bar{M}_{\mathrm{CHAB}}=0.08 \mathrm{M}_{\odot}\right)$.

\subsection{Initial mass function}

The stellar IMF gives the probability that a newly formed star has a certain mass (Chabrier 2003). The IMFs observed in different local star-forming regions appear to be very similar, implying that the star formation process is independent of environment (Kroupa 2001, 2002). Numerical simulations reproduce this universal IMF well for a large variety of initial conditions (Bate 2005, 2009a,b).

We cannot attempt to reproduce the observed IMF here because we have only treated a single core mass $\left(1 \mathrm{M}_{\odot}\right)$. Observed cores are known to have a distribution of masses, given by the core mass function (CMF), and the CMF appears to be similar in shape to the IMF but shifted to higher masses (e.g. André et al. 2010; Könyves et al. 2015, 2020). However we can evaluate the mean stellar mass function $(\mathrm{MF})$ produced by a $1 \mathrm{M}_{\odot}$ core, with and without outflow feedback. Fig. 4 shows the Chabrier IMF (solid black line), the cumulative MF for the OF-sample (solid orange line), the cumulative MF for the RF-sample (solid blue line), a lognormal fit to the OF-sample (dashed orange line) and a lognormal fit to the RF-sample (dashed blue line). The fits are obtained using data-likelihood maximization Markov-Chain Monte Carlo sampling. The mean masses, $\bar{M}_{\mathrm{CHAB}}=0.08 \mathrm{M}_{\odot}, \bar{M}_{\mathrm{OF}}=0.13 \mathrm{M}_{\odot}$ and $\bar{M}_{\mathrm{RF}}=$
$0.18 \mathrm{M}_{\odot}$ are shown as vertical dotted lines. The corresponding standard deviations are $\sigma_{\mathrm{CHAB}}=0.69 \pm 0.05, \sigma_{\mathrm{OF}}=0.40 \pm 0.06$ and $\sigma_{\mathrm{RF}}=0.44 \pm 0.06$. Thus, outflow feedback reduces the mean stellar mass produced by a $1 \mathrm{M}_{\odot}$ core by $\sim 28$ per cent (cf. Krumholz, Klein \& McKee 2012; Hansen et al. 2012). If we compare the cumulative MFs using the KS test, it returns statistics $s=0.24$ and $p<0.1$ per cent. We conclude that the OF- and the RF-samples are not drawn from the same underlying distribution. This conclusion is confirmed by an Anderson-Darling test (Stephens 1974).

If the fragmentation of a core into stars is a statistically self-similar process - in the sense that the probability that a core of mass $M_{\mathrm{CORE}}$ spawns a star of mass $M_{\star}$ is the same as the probability that a core of mass $\beta M_{\text {CORE }}$ spawns a star of mass $\beta M_{\star}$ - the width of the observed $\mathrm{IMF}$ is

$\sigma_{\mathrm{CHAB}} \simeq \sqrt{\sigma_{\mathrm{CORE}}^{2}+\sigma_{\mathrm{FRAG}}^{2}}$.

Here, $\sigma_{\mathrm{CORE}}$ is the logarithmic standard deviation of the CMF, and $\sigma_{\mathrm{FRAG}}$ is the logarithmic standard deviation of the stellar MF from a single core. Equation (15) implicitly assumes that both the CMF, and the stellar MF from a single core, are approximately lognormal. Substituting $\sigma_{\mathrm{FRAG}}=\sigma_{\mathrm{OF}}$, we obtain

$\sigma_{\mathrm{CORE}} \simeq \sqrt{\sigma_{\mathrm{CHAB}}^{2}-\sigma_{\mathrm{OF}}^{2}} \simeq 0.57 \pm 0.07$.

In other words - if the assumption of statistically self-similar core fragmentation is correct - the CMF makes a larger contribution to the standard deviation of the IMF than the process of core fragmentation. However, we should be mindful that the fragmentation of more massive cores might be very different from those we have simulated here.

\section{MULTIPLICITY}

Most field stars with $M_{\star} \gtrsim \mathrm{M}_{\odot}$, and a high fraction of those with lower mass, are in multiple systems (e.g. Raghavan et al. 2010; Whitworth \& Lomax 2015). The fraction of newly formed stars in multiple systems is even higher, and the presumption is that some of these multiples are subsequently ionized by $\mathrm{N}$-body interactions or tidal stresses to produce the distribution in the field. It follows that numerical simulations of star formation should (a) reproduce the multiplicity statistics observed, and (b) demonstrate how multiple systems actually form. 
We use three multiplicity descriptors (e.g. Reipurth \& Zinnecker 1993). The multiplicity frequency,

$m f=\frac{B_{2}+T_{3}+Q_{4}+Q_{5}+\ldots}{S_{1}+B_{2}+T_{3}+Q_{4}+Q_{5}+\ldots}$,

gives the number of systems with more than one member (i.e. order higher than one). The higher-order frequency,

$h f=\frac{T_{3}+Q_{4}+Q_{5}+\ldots}{S_{1}+B_{2}+T_{3}+Q_{4}+Q_{5}+\ldots}$.

gives the number of systems with more than two members (i.e. order higher than two). The pairing factor,

$p f=\frac{B_{2}+2 T_{3}+3 Q_{4}+4 Q_{5}+\ldots}{S_{1}+B_{2}+T_{3}+Q_{4}+Q_{5}+\ldots}$,

gives the average number of companions to a randomly picked primary star.

\subsection{VANDAM survey}

We compare the multiplicity statistics from our simulations with those from the VANDAM survey (Tobin et al. 2016), which used the VLA to measure the multiplicity statistics of 64 Class 0/I multiple protostars with separations between $15 \mathrm{AU}$ and 10,000 AU, in the Perseus molecular cloud. A proper comparison would require the generation of synthetic observations, taking account of sensitivity, beam size, UV-coverage, confusion and projection; for example, some of the close binary systems in our simulations have very small separations and might not be detectable as binaries. However, generating synthetic observations is outside the scope of this paper, and therefore we simply make direct comparisons between our simulations and the observations.

The protostars observed within the VANDAM survey are slightly more massive than the stars in our OF-sample. Tobin et al. (2016) do not provide masses for individual observed stars or multiple systems. However, using the protostellar luminosity function of McKee \& Offner (2010), McKee \& Offner (2011) they compute a protostellar mass function and expect their stars to be progenitors of $\mathrm{K}$ - and $\mathrm{M}$ dwarfs $\left(0.08-0.8 \mathrm{M}_{\odot}\right)$ with a mean protostellar mass of $\sim 0.2 \mathrm{M}_{\odot}$ of which $\sim 14$ per cent have masses between $0.7 \mathrm{M}_{\odot}$ and $2.5 \mathrm{M}_{\odot}$. In our simulations the mean protostellar mass at $t_{5}$ is $0.17 \pm 0.15 \mathrm{M}_{\odot}$ (OF-sample) and $0.26 \pm 0.25 \mathrm{M}_{\odot}$ (RF-sample), respectively. Only 3 per cent (OF-sample) and 5 per cent (RF-sample) of the stars are more massive than $0.7 \mathrm{M}_{\odot}$. Fig. 5 shows the stellar bolometric luminosity distribution of the multiple systems in our simulations at $t_{5}$. The luminosities are computed using the stellar evolution model by Offner et al. (2009; Sections 2.4 and 2.5). Since, at this point, most of the gas is either bound in stars or entrained by the outflows, we expect the stellar bolometric luminosity to be comparable with those observed by Tobin et al. (2016). Comparing the luminosities of multiple systems in our simulations to the VANDAM survey (Fig. 5) indicates that we are not probing exactly the same mass range. Despite this difference, the VANDAM survey is still the best survey of protostellar multiple systems and the only one we can compare our simulation with.

\subsection{Multiplicity statistics}

Fig. 6 shows the fractions of systems that are single, binary, triple, etc., for the OF-sample (orange) and the RF-sample (blue), at $t_{0.5}$ (top panel) and $t_{5}$ (middle and bottom panels), compared with the VANDAM survey (grey). The top and middle panels show the fractions

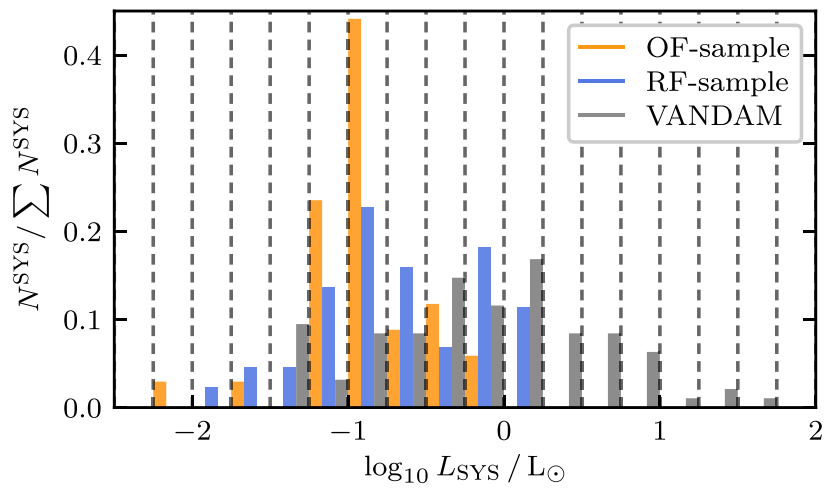

Figure 5. Stellar bolometric luminosities of all multiple systems in the OF-sample (orange) and RF-sample (blue) at $t_{5}$ compared to the observed bolometric luminosities of multiples in the VANDAM survey (grey; Tobin et al. 2016). The luminosities of the VANDAM survey multiples are higher, suggesting that somewhat more massive stars are present.

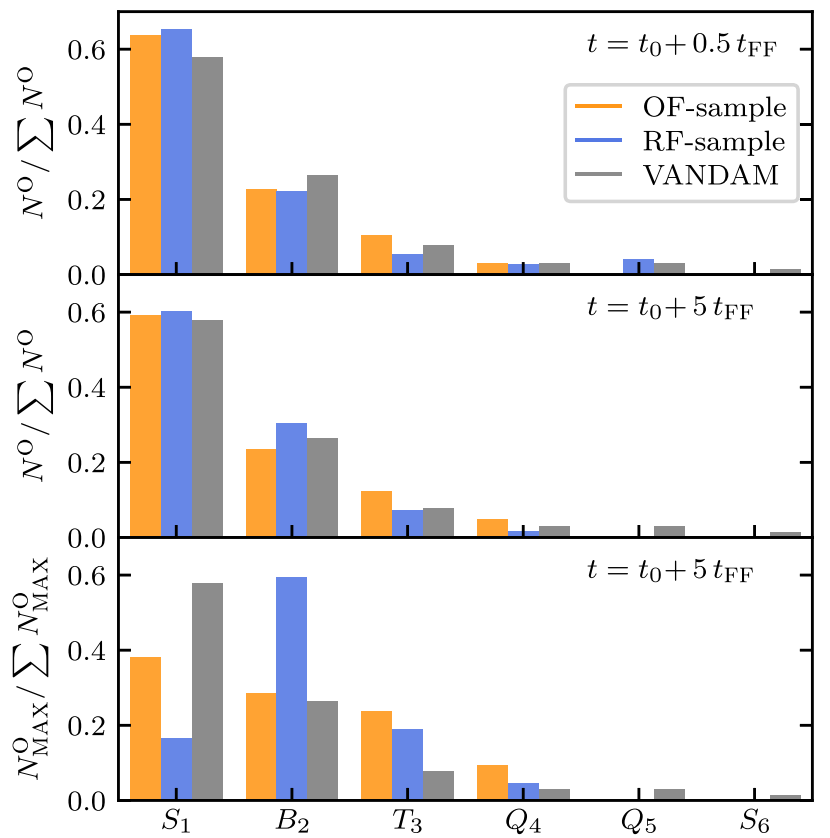

Figure 6. The fractions of systems that are single, binary, triple, etc., for the OF-sample (orange) and the RF-sample (blue), at $t_{0.5}$ (top panel) and $t_{5}$ (middle and bottom panels), compared with the VANDAM survey (grey). The top and middle panels include all systems, whereas the bottom panel includes only the highest-order system from each simulation. The OF-sample distribution changes little between the top and middle panels. In contrast, many of the quadruple and quintuple systems that form early in the RF-sample (top panel) quickly decay into binaries (middle panel); this is even clearer in the bottom panel where binaries dominate the distribution of highest-order systems for the RF-sample.

of all systems, $N^{O} / \sum^{O}\left\{N^{O}\right\}$, with $N^{O}$ the number of systems of order $O\left(=S_{1}, B_{2}, T_{3}, Q_{4}, Q_{5}\right.$, etc.). The bottom panel instead shows the distribution of $N_{\mathrm{MAX}}^{O} / \sum^{O}\left\{N_{\mathrm{MAX}}^{O}\right\}$, where $N_{\mathrm{MAX}}^{O}$ only takes account of the highest-order system from each simulation (Section 3.2).

Already by $t_{0.5}$ (Fig. 6, top panel) the RF-sample includes many HOMs, including quintuples. However, these systems are very unstable, and by $t_{5}$ (Fig. 6, middle panel) they have decayed to binaries. When taking into account only the highest-order systems, binaries dominate the distribution (Fig. 6, bottom panel). 


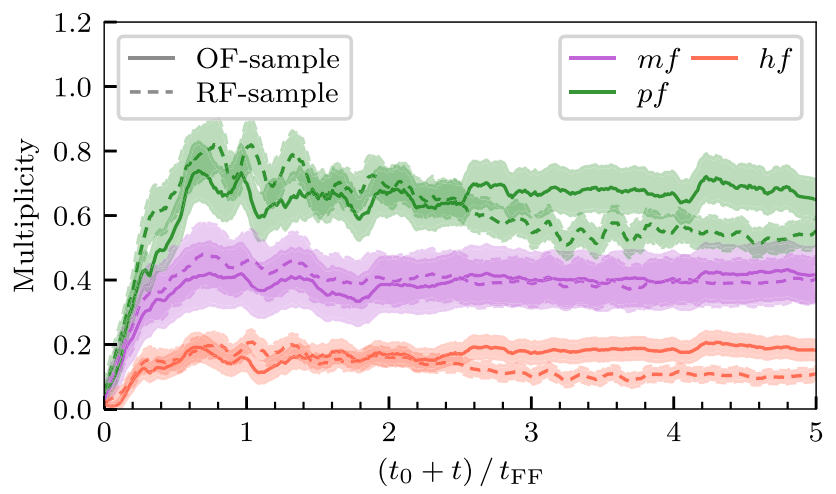

Figure 7. Time evolution of the multiplicity frequency, $\mathrm{mf}$ (purple), pairing factor, pf (green), and higher-order frequency, $\mathrm{hf}$ (red), for the OF-sample (solid lines) and RF-sample (dashed lines). The areas, surrounding the lines in corresponding colours, indicate the time-dependent propagated upper-limit on the Poisson uncertainty. The $x$-axis shows the time after the formation of the first sink, in units of the free-fall time. While the multiplicity frequency is comparable for the two samples, the pairing factor and higher-order frequency are significantly higher for the OF-sample after $2.5 t_{\mathrm{FF}}$.

In contrast, the multiplicity distribution of the OF-sample remains rather constant between $t_{0.5}$ and $t_{5}$. It is a monotonically decreasing function of the order, and matches the VANDAM survey well; the highest-order systems are not dominated by binaries.

The fraction of singles that are the highest-order system is low (Fig. 6, bottom panel) because many singles are low-mass stars that are ejected during the dynamical interactions that reduce HOMs to binary systems. This is particularly true for the RF-sample, where 89 per cent of singles are ejecta; for the OF-sample only 67 per cent of singles are ejecta.

\subsection{Time evolution of the stellar multiplicity}

Fig. 7 shows the time evolution of the multiplicity descriptors defined in equations (17) through (19), for all the simulations in the OFand RF-samples. Here, time is measured from when the first sink forms $\left(t_{0}\right)$, in units of the free-fall time of the birth core $\left(t_{\mathrm{FF}}\right)$. Once star formation starts, the multiplicity rises rapidly up to $\sim 0.6 t_{\mathrm{FF}}$. Thereafter the multiplicity frequency is approximately constant and comparable for both samples, $m f_{\mathrm{OF}} \sim m f_{\mathrm{RF}} \sim 0.40$. For the OFsample, most of the multiple systems are quite stable, and therefore the higher-order frequency and pairing factor are also approximately constant, at $h f_{\mathrm{OF}} \sim 0.15$ and $p f_{\mathrm{OF}} \sim 0.65$ respectively. However, for the RF-sample, the HOMs immediately start to eject lower-mass members and decay to binaries; this has no effect on the multiplicity frequency, $m f_{\mathrm{RF}}$, but the higher-order frequency and pairing factor both decrease steadily, and by $t \sim 5 t_{\mathrm{FF}}$, they are $h f_{\mathrm{RF}} \sim 0.10$ and $p f_{\mathrm{RF}} \sim 0.50$.

The multiplicities of the OF- and RF-samples are very similar in the early phase of star formation. The RF-sample forms somewhat more multiples and forms them somewhat faster, but these differences are small, and most of the HOMs formed in the RF-sample quickly reduce to binaries. HOMs are more stable against disruption when outflows are present.

Table 4 compares $\mathrm{mf}, \mathrm{pf}$, and hf for both samples at $t_{5}$ with the VANDAM survey. All the statistics for the OF-sample agrees with the VANDAM survey within the uncertainties; $m f_{\text {OF }}$ and $h f_{\text {OF }}$ agree very well, but $p f_{\mathrm{OF}}$ only just agrees. For the RF-sample, only $m f_{\mathrm{RF}}$ agrees with VANDAM, both $p f_{\mathrm{RF}}$ and $h f_{\mathrm{RF}}$ are much too low. This is largely due to the decay of HOMs in the RF-sample.
Table 4. Multiplicity statistics for the OF- and RF-samples at $t_{5}$, compared with the VANDAM survey (Tobin et al. 2016). The first column gives the sample, followed by the multiplicity frequency (equation 17), the pairing factor (equation 19), and the high-order frequency (equation 18).

\begin{tabular}{lccc}
\hline Sample & $\mathrm{mf}$ & $\mathrm{pf}$ & $\mathrm{hf}$ \\
\hline RF-sample & $0.40 \pm 0.07$ & $0.51 \pm 0.05$ & $0.09 \pm 0.02$ \\
OF-sample & $0.40 \pm 0.09$ & $0.63 \pm 0.06$ & $0.17 \pm 0.03$ \\
VANDAM & $0.40 \pm 0.06$ & $0.71 \pm 0.06$ & $0.16 \pm 0.04$ \\
\hline
\end{tabular}

Note that a direct comparison with the VANDAM survey is biased since they observe slightly more massive stars than we produce in our simulations (Section 4.1). Observations show that the multiplicity fraction is strongly dependent on the primary mass (see, e.g. Fig. 1 in Whitworth \& Lomax 2015). However, this relation is valid for mainsequence stars and it is not clear whether it holds for the pre-mainsequence regime. Taking this relation into account, it seems that our simulations produce a too high multiplicity, given the low protostellar masses. However, our simulations do not include magnetic fields. With magnetic fields, we would expect less fragmentation and hence a somewhat lower multiplicity (see the discussion in Section 4.6). The missing magnetic fields could possibly explain why we are matching the VANDAM survey so well, even though we are probing a lower mass regime.

\subsection{Stability of triple systems}

Fig. 7 shows that the fraction of HOMs in the RF-sample decreases after $t_{1}$, while the fraction is almost constant for the OF-sample. To confirm objectively that this is because the hierarchical triple systems in the OF-sample are more stable, we compute the two different criteria for the stability of hierarchical triple systems which Zhuchkov, Kiyaeva \& Orlov (2010) has shown to be most reliable.

An hierarchical triple system is one in which a pair of stars (labelled individually ' 1 ' and ' 2 ', and together ' $1+2$ ') are on a tight orbit around one another, and this pair and a third star (labelled ' 3 ') are then on a much wider orbit around one another. The masses of the stars are $m_{1}, m_{2}$, and $m_{3}$, and the total mass of the tight pair is $m_{\mathrm{IN}}=m_{1}+m_{2}$. The semimajor axis and eccentricity of the tight orbit (involving stars 1 and 2) are $a_{\mathrm{IN}}$ and $e_{\mathrm{IN}}$. The semimajor axis and eccentricity of the wide orbit (involving star 3 and the pair 1+2) are $a_{\text {OUT }}$ and $e_{\text {OUT }}$. The system is hierarchical in the sense that $a_{\text {OUT }} \gg a_{\mathrm{IN}}$. With these definitions, the criterion for stability developed by Aarseth (2003) is

$f_{\mathrm{A}}=0.36 \frac{a_{\mathrm{OUT}}\left(1-e_{\mathrm{OUT}}\right)}{a_{\mathrm{IN}}}\left[\left(1+\frac{m_{3}}{m_{\mathrm{IN}}}\right) \frac{1+e_{\mathrm{OUT}}}{\sqrt{1-e_{\mathrm{OUT}}}}\right]^{-2 / 5}>1 ;$

and the criterion developed by Valtonen, Karttunen \& Gutzwiller (2007) is

$$
\begin{aligned}
f_{\mathrm{V}}= & 3 \frac{a_{\mathrm{OUT}}\left(1-e_{\mathrm{OUT}}\right)^{7 / 6}}{a_{\mathrm{IN}}} \\
& \times\left[\left(1+\frac{m_{3}}{m_{\mathrm{IN}}}\right)\left(\frac{7}{4}+\frac{\cos (i)}{2}-\cos ^{2}(i)\right)\right]^{-1 / 3}>1,
\end{aligned}
$$

where $i$ is the angle between the angular momentum vectors of the tight and wide orbits.

Fig. 8 shows the time evolution of $\bar{f}_{\mathrm{A} . \mathrm{OF}}$ (orange dashed line) and $\bar{f}_{\mathrm{V} \text {.OF }}$ (orange full line), i.e. $f_{\mathrm{A}}$ and $f_{\mathrm{V}}$ averaged over all the triple systems in the O-sample; and the time evolution of $\bar{f}_{\mathrm{A} \text {.RF }}$ (blue dashed line) and $\bar{f}_{\mathrm{V} . \mathrm{RF}}$ (blue full line), i.e. $f_{\mathrm{A}}$ and $f_{\mathrm{V}}$ averaged over all the triple systems in the R-sample. $\bar{f}_{\mathrm{A} . \mathrm{OF}}$ is almost indistinguishable from 


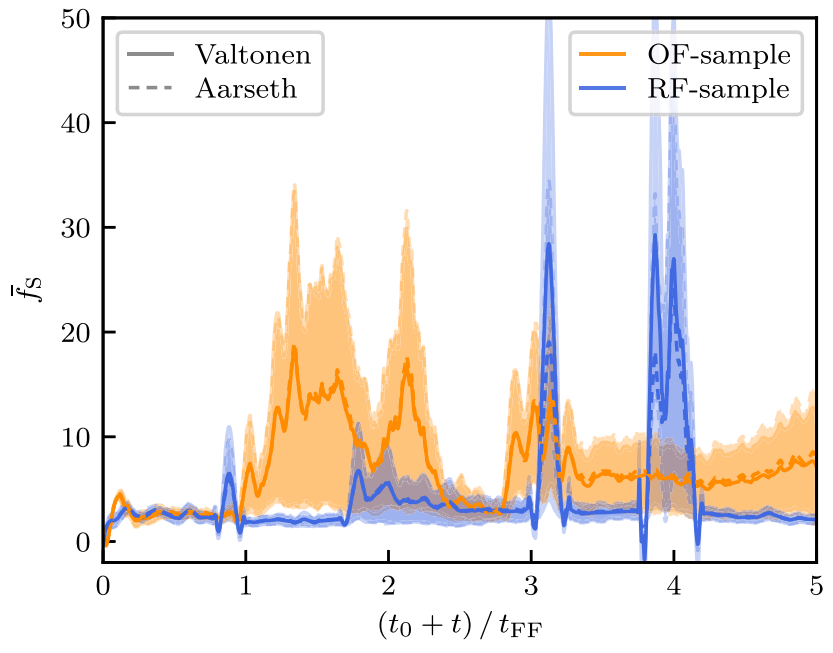

Figure 8. Time evolution of the Aarseth and Valtonen stability parameters (equations 20 and 21), averaged over all the triple systems in the OF-sample (orange), and over all the triple systems in the RF-sample (blue). The dashed lines show the results obtained for the Aarseth parameter $\left(f_{\mathrm{A}}\right)$, and the solid lines show the results obtained for the Valtonen parameter $\left(f_{\mathrm{V}}\right)$. At most times the dashed lines cannot be discerned because they sit on top of the solid lines. The coloured shading represents the standard deviation about the mean. Triple systems in the OF-sample are markedly more stable than triple systems in the RF-sample.

$\bar{f}_{\text {V.OF }}$, and likewise $\bar{f}_{\text {A.RF }}$ from $\bar{f}_{\text {V.RF }}$, indicating that they are mutually consistent. After $t_{\mathrm{FF}}, \bar{f}_{\mathrm{A} . \mathrm{OF}}$, and $\bar{f}_{\mathrm{V} \text {.OF }}$ are almost always well above $\bar{f}_{\text {A.RF }}$ and $\bar{f}_{\text {V.RF }}$, on average by a factor $2.53 \pm 0.05 . \bar{f}_{\text {A.OF }}$ and $\bar{f}_{\text {V.OF }}$ are also well above unity most of the time, and end up at $\sim 7$, so most of the triples are stable. In contrast, $\bar{f}_{\mathrm{A} . \mathrm{RF}}$ and $\bar{f}_{\mathrm{V} . \mathrm{RF}}$ are almost always $\sim 2$, so outliers with lower than average values tend to be unstable and decay.

\subsection{Twin binaries}

Observations of low-mass binary systems reveal a high fraction of systems in which the ratio of the secondary mass to the primary mass, $q=M_{\star-\mathrm{S}} / M_{\star-\mathrm{P}}$, is close to unity (see Lucy 2006; Simon \& Obbie 2009; Fernandez et al. 2017; Kounkel et al. 2019; El-Badry et al. 2019, or the review by Duchêne \& Kraus (2013)); these systems are referred to as 'twin binaries'. Fig. 9 shows the distribution of $q$ for the 35 binary systems in the OF-sample, and the 44 binaries in the RF-sample. Both samples are peaked at high $q$ with a tail towards low $q$, similar to the observations reported by Fernandez et al. (2017) and Kounkel et al. (2019). The convention (e.g. Kounkel et al. 2019; El-Badry et al. 2019) is to class binary systems with $q \geq q_{\text {CRIT }}=$ 0.95 as twins. Using this definition, $f_{\mathrm{TWIN}-\mathrm{OF}}=43$ per cent for the OF-sample, compared with $f_{\text {TWIN-RF }}=9$ per cent for the RF-sample. Since, with the small number of binaries in our sample, the ratio $f_{\text {TWIN-OF }} / f_{\text {TWIN-RF }}$ is particularly high for $q_{\mathrm{CRIT}}=0.95$, we have varied $q_{\text {CRIT }}$ between 0.90 and 0.99 , but for all $q$-values in this range, there are always more twins in the OF-sample, $f_{\text {TWIN-OF }} / f_{\text {TWIN-RF }} \geq 2.1 \pm 0.6$.

The excess of almost equal mass binaries is thought to be the result of competitive accretion (Tokovinin 2000). For a low- $q$ binary, the secondary is on a larger orbit and therefore accretes matter with high specific angular momentum faster than the primary, thereby driving the mass ratio towards unity (Whitworth et al. 1995; Young \& Clarke 2015; Matsumoto, Saigo \& Takakuwa 2019). In the present context this happens because the binary is accreting from a circumbinary disc (see Fig. 1 and El-Badry et al. 2019).

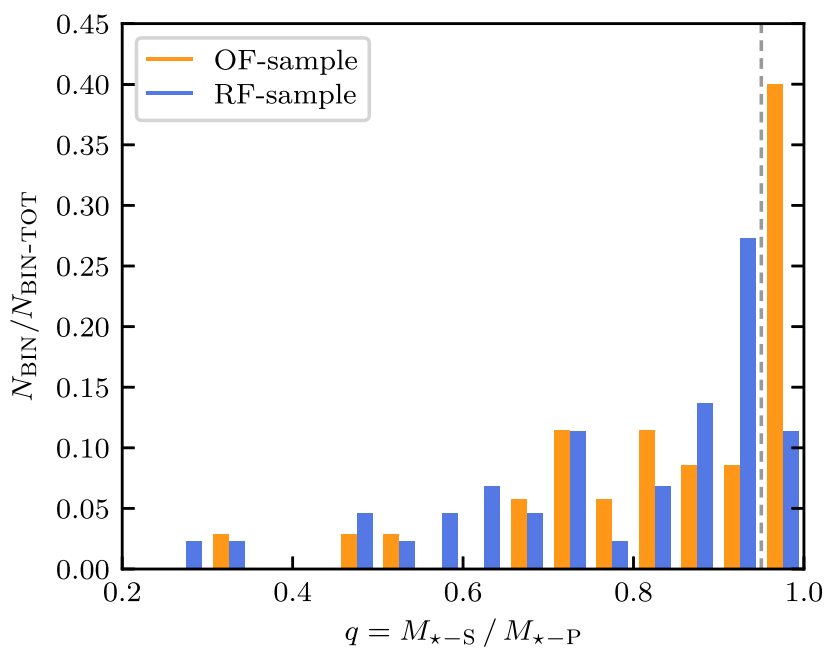

Figure 9. The normalized distribution of the mass ratio between the secondary and primary components, $q=M_{\star-\mathrm{S}} / M_{\star-\mathrm{P}}$, for all binary systems in the OF-sample (orange) and all binary systems in the RF-sample (blue), at $t_{5} \equiv t_{0}+5 t_{\mathrm{FF}}$. At this stage there are 35 binaries in the OF sample, and 44 in the RF-sample. Both distributions are peaked at high $q$, with a tail towards low $q$. Outflow feedback appears to enhance the formation of twin binaries with $q \geq 0.95$ (bin to the right of the dashed grey line).

Fig. 10 shows the two-dimensional probability density function (PDF) for the stellar mass $M_{\star}$ at $t_{5}$ and the fraction of this mass acquired by disc accretion as opposed to direct infall, $f_{\text {DISC }}=$ $M_{\text {DISC }} /\left(M_{\text {DISC }}+M_{\text {DIRECT }}\right)$. The corresponding one-dimensional PDFs are shown in the top and right-hand panels.

To compute $f_{\text {DISC }}$ we evaluate the mass flow through a sphere around each star, with the radius of the sphere equal to half the radius of the corresponding accretion disc. SPH particles are assigned to an accretion disc if (a) their density is $>10^{-14} \mathrm{~g} \mathrm{~cm}^{-3}$, (b) their rotational velocity component is greater than their radial velocity component with respect to the corresponding sink, and (c) they are gravitationally bound to the star. The mean disc radius for the OFsample at $t_{2}$ is $\bar{r}_{\text {DISC }}=103 \pm 55 \mathrm{AU}$, which is in good agreement with recent observations of Class 0/I stars (Maury et al. 2019). Only stars in multiple systems that have both an accretion disc and a velocity lower than the escape speed contribute to the evaluation of $f_{\text {DISC }}$. The mass flow contributes to $M_{\mathrm{DISC}}$ if $\frac{\pi-\theta_{\mathrm{OPEN}}}{2}<\theta<\frac{\pi+\theta_{\text {OPEN }}}{2}$, where $\theta$ is the angle between the position vector (relative to the star) and the angular momentum axis of the disc. We set $\theta_{\mathrm{OPEN}}=1 / 3 \pi$; varying $\theta_{\text {OPEN }}$ between $1 / 2 \pi$ and $1 / 6 \pi$ shows no qualitative difference.

The peak of the OF-sample PDF is shifted to higher $f_{\text {DISC }}$ compared with the peak of the RF-sample. A one-sided Mann-Whitney-U test confirms that this difference is significant with $p \ll 1$ per cent Outflow cavities stop stars from accreting so rapidly via direct infall, thereby enhancing the contribution from disc accretion and hence increasing the fraction of twin binaries in the OF-sample.

\subsection{Magnetic fields and disc fragmentation}

Observations show that dense cores are threaded by magnetic fields (Troland \& Crutcher 2008; Kandori et al. 2018). Recent numerical simulations suggest that magnetic fields have a profound impact on the star formation process (see, e.g. the review of Wurster \& $\mathrm{Li}$ 2018). In the ideal MHD case, magnetic braking almost entirely prohibits the formation of accretion discs (Commerçon et al. 2012; Bate et al. 2014). However, the introduction of non-ideal MHD 

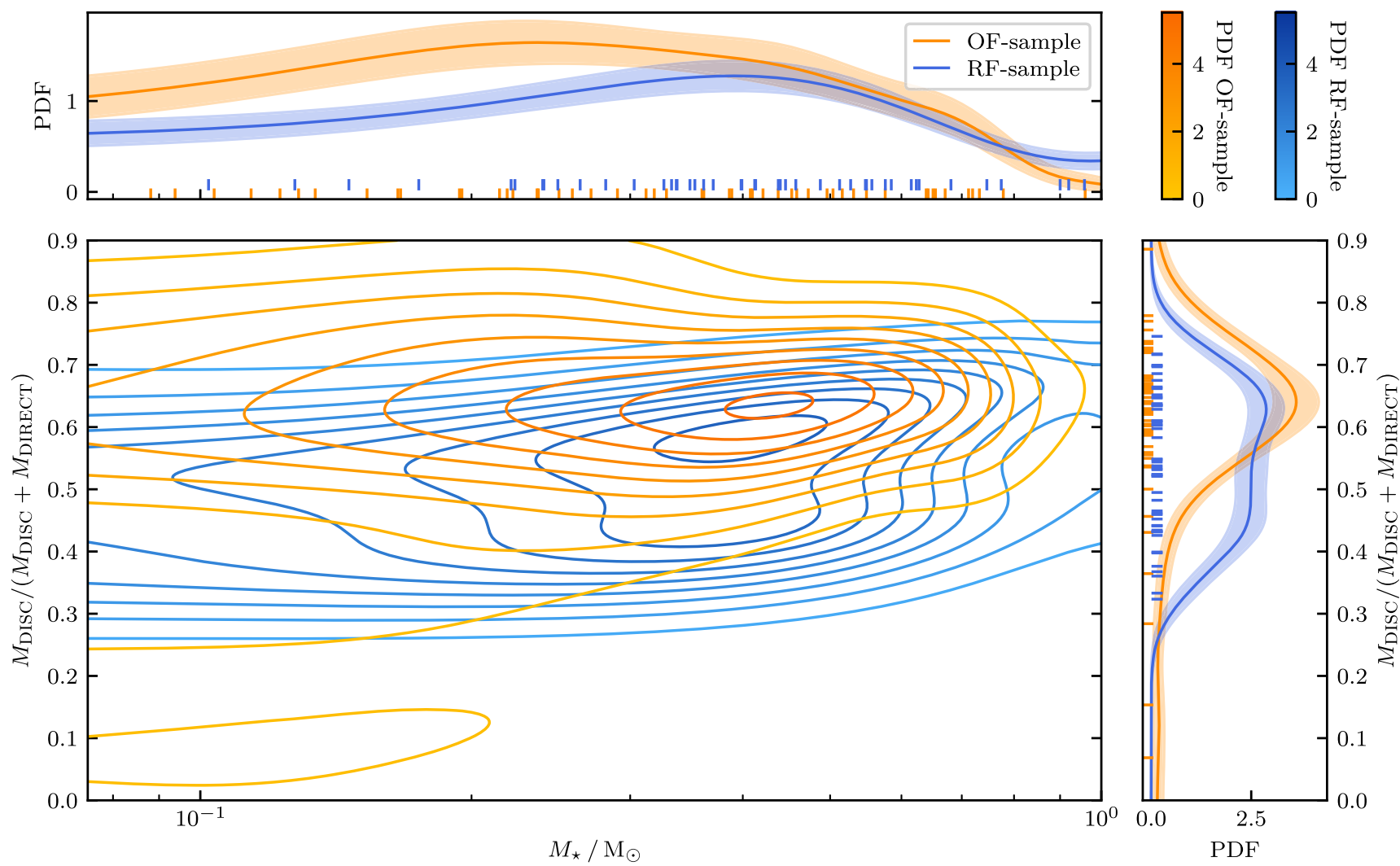

Figure 10. The two-dimensional probability density function (PDF) for the 'final' stellar mass, $M_{\star}$, against the fraction of that mass that has been acquired by disc accretion (as opposed to by direct infall), $M_{\mathrm{DISC}} /\left(M_{\mathrm{DISC}}+M_{\mathrm{INFALL}}\right)$; here 'final' means at $t_{5} \equiv t_{0}+5 t_{\mathrm{FF}}$. Contours at 10 per cent, 20 per cent, $\ldots . .90$ per cent of the maximum probability are in shades of orange for the OF sample, and in shades of blue for the RF sample. The top panel shows the one-dimensional PDF for $M_{\star}$. The right-hand panel shows the one-dimensional PDF for $M_{\text {DISC }} /\left(M_{\text {DISC }}+M_{\text {INFALL }}\right)$. On the top and right-hand panels, the shaded regions indicate uncertainties estimated using bootstrapping, and the small tick marks represent individual systems. Evidently simulations with outflow feedback lead to stars accreting more gas by disc accretion.

effects mitigates the efficiency of magnetic braking to some extent (Hennebelle et al. 2016; Wurster, Price \& Bate 2016; Zhao et al. 2018). Moreover, turbulence can cause a misalignment of the angular momentum and the magnetic field vector, which may significantly reduce the magnetic braking efficiency and enable the formation of massive discs (Seifried et al. 2013, 2015; Wurster et al. 2016; Gray, McKee \& Klein 2018; Wurster \& Li 2018; Wurster et al. 2019). Including the Hall-effect in non-ideal MHD simulations, Wurster \& Li (2018) show that for the case of anti-aligned magnetic field and angular momentum vectors, they obtain results which are most similar to a pure hydrodynamical calculation. The magnetic Toomre$Q$ parameter implies that these magnetized discs are generally more stable against fragmentation than pure hydrodynamical discs (Toomre 1964; Kim \& Ostriker 2001; Wurster \& Bate 2019).

Our code, GANDALF, does not currently include magnetic fields. With magnetic fields, we would expect to have slightly smaller discs that fragment less readily (Hennebelle \& Inutsuka 2019; Wurster \& Bate 2019). Therefore, the number of simulations forming a single star would increase resulting in fewer ejected stars and somewhat lower multiplicities.

Compared to the RF-sample, our OF-sample contains significantly more cores which form only a single star (Section 3.2). With episodic accretion feedback, the accretion discs are frequently severely disturbed and the amount of fragmentation is damped to a realistic level (Stamatellos et al. 2012; Lomax et al. 2014, 2015; Mercer \& Stamatellos 2017). We therefore speculate that magnetic fields would have a limited additional effect on disc fragmentation if episodic accretion feedback were taken into account.

\section{SELF-REGULATION OF OUTFLOW FEEDBACK}

\subsection{Entrainment factor}

Molecular outflows from protostars consist mainly of secondary entrained material (Tabone et al. 2017; Zhang et al. 2019), i.e. core gas that is swept up by the primary ejected gas. For low-mass star formation the entrained gas mass is estimated to range from $0.1 \mathrm{M}_{\odot}$ to $1.0 \mathrm{M}_{\odot}$ (Arce et al. 2007). The entrainment factor $\epsilon_{\mathrm{OF}}$ is defined as the ratio of total outflowing mass, $M_{\mathrm{OuT}}$, to primary ejected mass, $M_{\text {EJECT }}$. An SPH particle contributes to $M_{\mathrm{OUT}}$ if its radial velocity is higher than the local escape velocity and at least $0.1 \mathrm{~km} \mathrm{~s}^{-1}$. Using numerical MHD simulations, Offner \& Chaban (2017) conclude that $\epsilon_{\mathrm{OF}} \sim 4$.

Fig. 11 shows the entrainment factors at $t_{0.5}$ (top row), $t_{1.5}$ (middle row), and $t_{5}$ (bottom row), plotted against the total stellar mass, $M_{\star \text { тотАL }}$ (left-hand column) and against outburst frequency, $f_{\mathrm{OB}}$ (righthand column). Most simulations have $\epsilon_{\mathrm{OF}} \sim 7$, but some have much higher values, up to $\epsilon_{\mathrm{OF}} \sim 33$, particularly at low $M_{\star \text { TOTAL }}$ and/or low $f_{\mathrm{OB}}$. By $t_{5}$ there is a well-defined anticorrelation between $\epsilon_{\mathrm{OF}}$ and $M_{\star \text { TOTAL }}$, with $5 \lesssim \epsilon_{\mathrm{OF}} \lesssim 26$ and Spearman Rank Correlation coefficient $r_{\mathrm{S}}=-0.94$ and $p \ll 0.01$ per cent. 


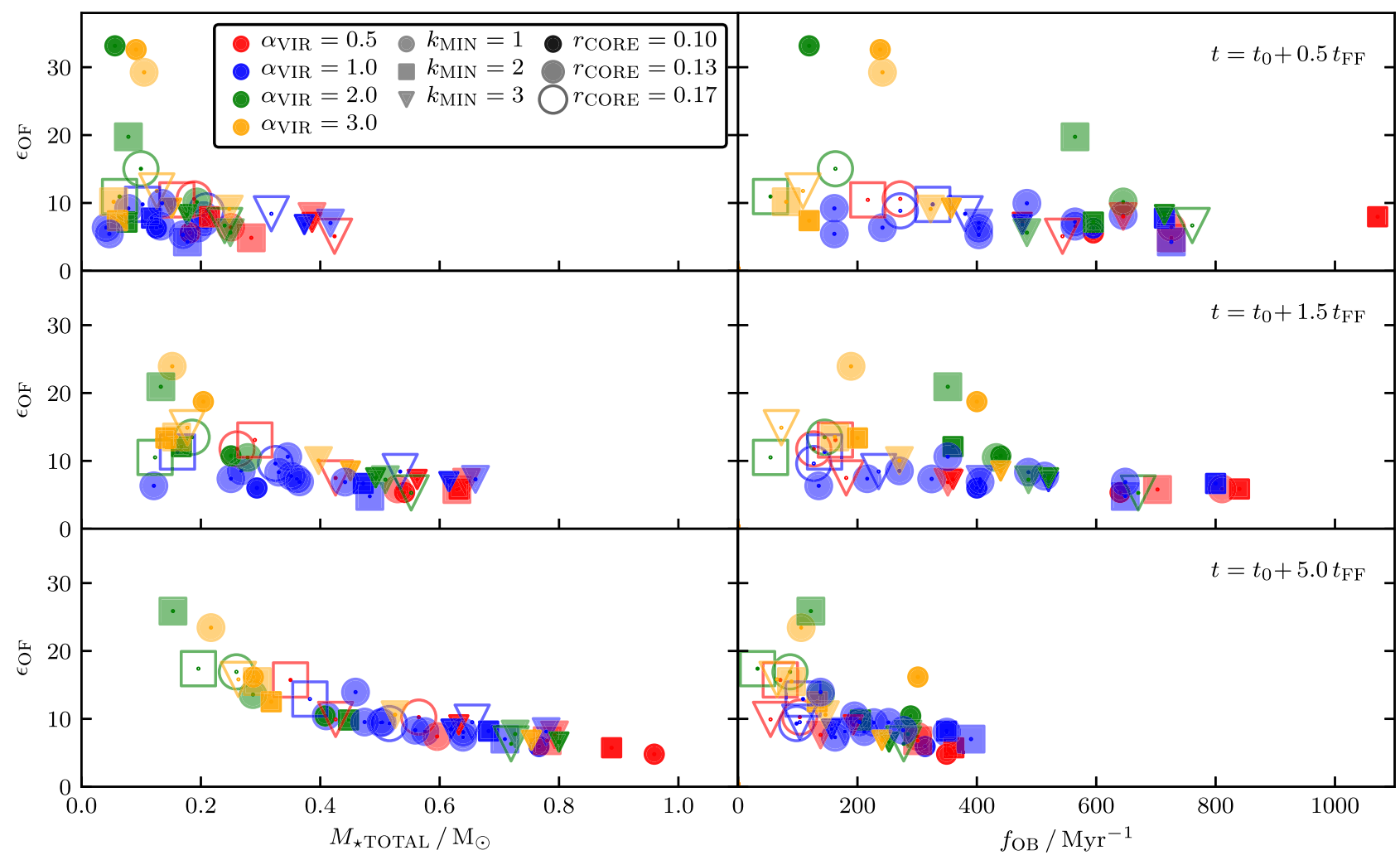

Figure 11. Entrainment factors, $\epsilon_{\mathrm{OF}}=M_{\mathrm{OUT}} / M_{\mathrm{EJECT}}$, for all the simulations in the OF sample, plotted against total stellar mass, $M_{\star \mathrm{TOTAL}}($ left-hand column), and against outburst frequency, $f_{\mathrm{OB}}$ (right-hand column), at $t_{0.5}$ (top row), $t_{1.5}$ (middle row), and $t_{5}$ (bottom row), where $t_{\tau}=t_{0}+\tau t_{\mathrm{FF}}$. At $t_{5}$, the entrainment factors range from $\epsilon_{\mathrm{OF}} \simeq 5$ to $\epsilon_{\mathrm{OF}} \simeq 26$. With increasing time, $\epsilon_{\mathrm{OF}}$ becomes strongly anticorrelated with $M_{\star \text { TOTAL }}$, and weakly anticorrelated with $f_{\mathrm{OF}}$.

There are several possible reasons for this anticorrelation. The higher the total mass in stars, the less mass there is left in the core envelope, and therefore the less mass there is left to entrain. Moreover, as time advances the outflows are increasingly likely to be launched into cavities blown by previous outflows, in which case there is even less material for them to entrain; this is especially true when a core has formed a multiple system with aligned outflows.

The right-hand column of Fig. 11, shows the entrainment factor, $\epsilon_{\mathrm{OF}}$, against the outburst frequency, $f_{\mathrm{OB}}$, averaged between the time when the first star forms, $t_{0}$, and - respectively $-t_{0.5}, t_{1.5}$, and $t_{5}$. There is an anticorrelation between $\epsilon_{\mathrm{OF}}$ and $f_{\mathrm{OB}}$ which gets stronger with time. By $t_{5}$, the Spearman rank correlation coefficient is $r=-0.70$ with $p \ll 0.01$ per cent. Simulations with lower $f_{\text {Ов }}$ (i.e. episodic accretion and outflow concentrated in a few massive outbursts) have higher $\epsilon_{\mathrm{OF}}$. The asymptotic limit of high $f_{\mathrm{OB}}$ is continuous outflow, and this might explain the lower $\epsilon_{\mathrm{OF}} \sim 4$ found by Offner \& Chaban (2017), since the protostars in their simulations generate continuous outflows.

\subsection{Outflowing gas mass}

Fig. 12 (right-hand column) shows the outflowing gas mass, $M_{\mathrm{OUT}}$, for each simulation in the OF-sample, plotted against its total stellar mass, $M_{\star \text { TOTAL }}$, at $t_{0.5}, t_{1.5}$ and $t_{5}$. At $t_{0.5}, M_{\text {OUT }}$ and $M_{\star \text { тотAL }}$ are tightly correlated, $M_{\text {OUT }} \simeq 0.01 \mathrm{M}_{\odot}+0.58 M_{\star \text { TOTAL }}$, with Spearman Rank Correlation coefficient $r=0.76$ and $p \ll 0.01$ per cent. Since 10 per cent of the matter entering a sink is ejected, this corresponds to an average entrainment factor of $\epsilon_{\mathrm{OF}} \sim 0.58 / 0.1 \sim 6.4$, in good agreement with Fig. 11.
At later times, the correlation remains strong but flattens, because cores with low $M_{\star \text { ТотАL }}$ have more gas left, and therefore higher entrainment factors and more massive outflows (see Fig. 11). By $t_{5}$, the correlation has become $M_{\text {oUT }} \simeq 0.29 \mathrm{M}_{\odot}+0.29 M_{\star \text { TOTAL }}$ with a non-zero intercept.

\subsection{Relative star formation efficiency}

Fig. 12 (left-hand column) shows the total stellar mass of each simulation in the OF-sample, $M_{\star \text { TотAL-OF }}$, plotted against the total stellar mass in the corresponding simulation in the RF-sample, $M_{\star \text { TOTAL-RF }}$, at $t_{0.5}, t_{1.5}$, and $t_{5}$. At $t_{0.5}$, the masses are strongly correlated, $M_{\star \text { TOTAL-OF }} \sim 0.60 M_{\star \text { TOTAL-RF }}$, with Spearman Rank Correlation coefficient $r=0.90$ and $p \ll 0.01$ per cent. Thus, the early star formation rate (SFR) is $\sim 40$ per cent lower if outflows are present.

This strong correlation arises because (a) $f_{\mathrm{EJECT}}=0.1$, i.e. exactly 10 per cent of the matter entering a sink is ejected and the remaining 90 per cent is accreted, so $\mathrm{d} M_{\star} /\left.\mathrm{d} t\right|_{\mathrm{OF}}=9 \mathrm{~d} M /\left.\mathrm{d} t\right|_{\mathrm{EJECT}}$; and (b) in the early stages the entrainment factor is approximately universal, $\epsilon_{\mathrm{OF}} \simeq 7$, so $\mathrm{d} M /\left.\mathrm{d} t\right|_{\mathrm{OUT}} \sim 7 \mathrm{~d} M /\left.\mathrm{d} t\right|_{\mathrm{EJECT}}$. It follows that

$$
\frac{\mathrm{d} M_{\star} /\left.\mathrm{d} t\right|_{\text {OF }}}{\mathrm{d} M_{\star} /\left.\mathrm{d} t\right|_{\text {OF }}+\mathrm{d} M /\left.\mathrm{d} t\right|_{\text {OUT }}} \sim \frac{9}{9+7}=56 \text { per cent. }
$$

If one assumes that, in the absence of outflow feedback, the outflowing matter would have ended up in the stars, the mass of stars in the OF-sample should be of order 56 per cent of the mass of stars in the RF-sample.

At later times the correlation persists, but with greater scatter. For example, at $t_{5}, M_{\star \text { TOTAL-OF }} \simeq 0.53 M_{\star \text { TOTAL-RF }}$ with Spearman Rank Correlation coefficient $r=0.65$ and $p<0.01$ per cent. 

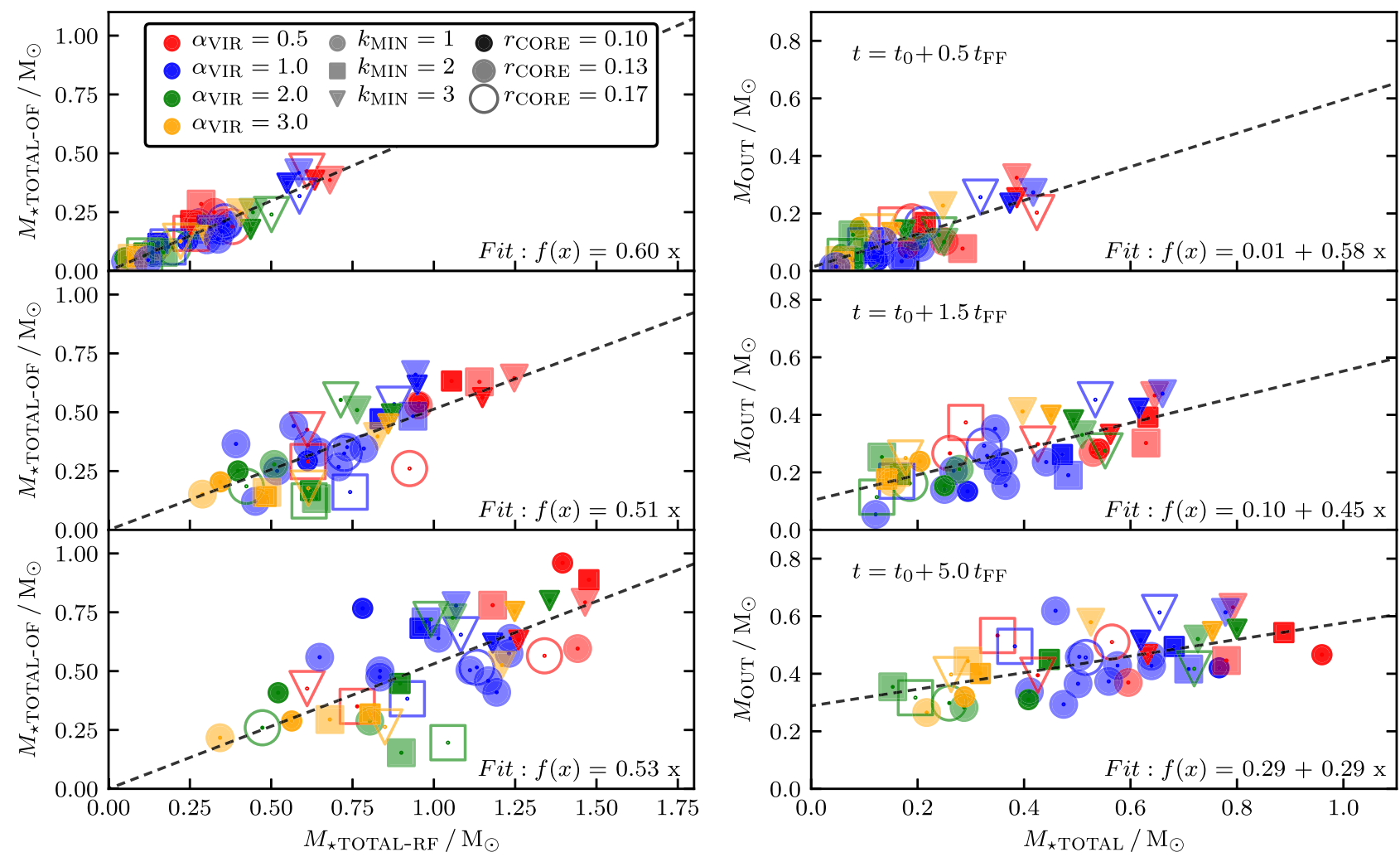

Figure 12. Left: The total stellar mass for each simulation in the OF-sample against the total stellar mass for the corresponding simulation in the RF sample, at $t_{0.5}$ (top), $t_{1.5}$ (middle), and $t_{5}$ (bottom), where $t_{\tau}=t_{0}+\tau t_{\mathrm{FF}}$. The dashed lines are linear fits to the data, with $0.5 \lesssim M_{\star \text { tOTAL-OF }} / M_{\star \text { TOTAL-RF }} \lesssim 0.6$. At $t_{0.5}$ the fit is very tight, but the scatter around the fit increases at later times, due to changes in the entrainment factor and hence changes in the effectiveness of outflow feedback (see Fig. 11). Right: The outflowing gas mass, $M_{\text {OUT }}$, for all the simulations in the OF sample, against the total stellar mass, $M_{\star \text { тотAL }}$, at $t_{0.5}$ (top), $t_{1.5}$ (middle), and $t_{5}$ (bottom). At $t_{0.5}$, there is a strong correlation, $M_{\text {OUT }} \simeq 0.01 \mathrm{M}_{\odot}+0.58 M_{\star \text { тотАL }}$. At later times, the correlation remains strong, but flattens, becoming $M_{\text {OUT }} \simeq 0.29 \mathrm{M}_{\odot}+0.29 M_{\star \text { TOTAL }}$ at $t_{5}$; this is because by this stage cores with relatively low $M_{\star \text { TOTAL }}$ have more gas left, and therefore higher entrainment factors and - relatively speaking - more massive outflows (see Fig. 11).

\subsection{Possible effects of magnetic fields on the outflow structure}

Magnetic fields are obviously very important for protostellar outflows since magnetic fields are necessary to launch outflows in the first place (Bally 2016). This regime is covered by our episodic outflow subgrid model, which is based on the episodic MRI instability of the inner disc. Computing the outflow launching selfconsistently is not expected to alter the outflow properties significantly with respect to our subgrid model (see, e.g. Federrath et al. 2014).

The influence of magnetic fields on the already launched or entrained gas is not well understood. Outflows are highly connected to the stellar accretion rate. Therefore, it is complicated to disentangle (i) the lower accretion rate due to magnetic fields (Offner \& Chaban 2017) from (ii) the direct effects of magnetic fields on the outflowing gas. We argue that (i) episodic outflows are highly self-regulated (Rohde et al. 2019) and that therefore a slower collapse would not alter the outflow properties notably. Offner \& Chaban (2017) show that magnetic fields do not affect the entrainment factor (Section 5.1) and therefore (ii) the influence on already launched gas is limited. Moreover, the typical velocity of outflowing gas, up to $\sim 100 \mathrm{~km} \mathrm{~s}^{-1}$, is much higher than the characteristic Alfvén speed, $v_{\mathrm{A}}=B / \sqrt{\rho}=1 \mathrm{~km} \mathrm{~s}^{-1}$ for typical values of $B=10 \mu \mathrm{G}$ and $\rho=10^{-20} \mathrm{~g} \mathrm{~cm}^{-3}$ for the low-density gas in the outflow cavity. Therefore, we argue that including magnetic fields would not alter the properties of the outflows in this work significantly.
However, in simulations of star cluster formation with initial gas masses of 100-1000 $\mathrm{M}_{\odot}$, it has been shown that the combination of outflows and magnetic fields is important. The momentum delivered by the outflows coupled to the magnetic fields maintains turbulence such that the parental molecular clouds stay close to virial equilibrium (see the review by Krumholz \& Federrath 2019). Therefore, we might not be able to expand our simulations to larger scales without taking into account the potentially significantly higher impact of magnetic fields.

\section{CONCLUSIONS}

Outflows are the dominant feedback mechanism in the early phase of low-mass star formation. However, the consequences of outflow feedback for the evolution of a pre-stellar core are not well understood. Three questions are especially important. (1) Does outflow feedback affect the properties of the individual stars formed? (2) Does outflow feedback affect the stellar multiplicity statistics? (3) How much does outflow feedback reduce the SFE?

To answer these questions, we have performed a large ensemble of smoothed particle hydrodynamics simulations of dense pre-stellar cores. All the cores have the dimensionless density profile of a Bonnor-Ebert sphere, but we vary the initial core radius $\left(r_{\mathrm{CORE}}=0.017,0.013,0.01 \mathrm{pc}\right)$, the initial virial ratio $\left(\alpha_{\mathrm{VIR}}=\right.$ $0.5,1.0,2.0,3.0)$, the wavenumber of the dominant mode in the initial turbulent velocity field $\left(k_{\mathrm{MIN}}=1,2,3\right)$. For each combination 
of $\left(r_{\mathrm{CORE}}, \alpha_{\mathrm{VIR}}, k_{\mathrm{MIN}}\right)$ and eight additional runs with different turbulent seeds, we perform one simulation with outflow feedback, and one without. The resulting ensemble of 88 simulations reveals the following features.

(i) The stellar statistics (total mass in stars, number of stars, mass of most massive star, total mass and order of the highest-order system) depend only weakly on the initial conditions of the birth core, i.e. $\left(r_{\mathrm{CORE}}, \alpha_{\mathrm{VIR}}, k_{\mathrm{MIN}}\right)$.

(ii) The total mass in stars, the mass of the most massive star and the total mass of the highest-order system all tend to decrease markedly when outflow feedback is included. The total number of stars and the order of the highest-order system tend to decrease very slightly when outflow feedback is included - except for the cores with low $\alpha_{\mathrm{VIR}}$ where these trends are reversed.

(iii) The distribution of stellar masses can be represented by a lognormal. Without outflow feedback the mean and standard deviation of $\log _{10}\left(M_{\star} / \mathrm{M}_{\odot}\right)$ are -0.74 and 0.44 . When outflow feedback is included, they become -0.89 and 0.40 , i.e. the mean mass is reduced by $\sim 30$ per cent.

(iv) The simulations without outflow feedback produce a large number of HOMs, but many of them are unstable and quickly decay to binaries. As a result, these simulations deliver an higher-order frequency (hf) and a pairing factor (pf) that are inconsistent with the values observed in the VANDAM survey by Tobin et al. (2016).

(v) The simulations with outflow feedback produce slightly fewer HOMs, but most of them are stable. As a result, these simulations deliver an $\mathrm{hf}$ and a pf which, within the uncertainties, agree with the distribution observed in the VANDAM survey by Tobin et al. (2016).

(vi) The inclusion of outflow feedback increases considerably the fraction of twin binaries with almost equal-mass components. This is because outflow feedback reduces the role of direct infall on to a growing protostar, so the components of a binary system have to acquire their mass by accretion from a circumbinary disc.

(vii) The mean entrainment factor (the ratio between outflowing mass at large radius and the mass ejected from the protostar and its disc) is $\bar{\epsilon}_{\mathrm{OF}} \sim 7$, significantly larger than the value of 4 obtained by Offner \& Chaban (2017) in simulations with continuous outflow. Above average $\epsilon_{\mathrm{OF}}$ values are confined to cores with low total stellar mass (especially those with high $\alpha_{\mathrm{VIR}}$ and low $r_{\mathrm{CORE}}$ ), because there is then more gas left to entrain (and it is more easily unbound).

(viii) In the early stages, the outflowing mass, $M_{\mathrm{OUT}}$, is approximately proportional to the total stellar mass, $M_{\text {OUT }} \sim 0.7 M_{\star \text { TотAL }}$. At later times this is still true in cores where $M_{\star \text { TOTAL }}$ is low, but in cores where $M_{\star \text { TотAL }}$ is high, and there is less gas left to push out, $M_{\text {OUT }}<0.7 M_{\star \text { TOTAL }}$.

(ix) Since we have assumed that the rate of mass ejection from a protostar is exactly proportional to the rate of mass accretion on to the protostar, and since there is not a huge variation in entrainment factors, the mass converted into stars when outflow feedback is included is an approximately constant fraction of the mass converted into stars when there is no outflow feedback; after five free-fall times this fraction is $\sim 53$ per cent. This is partly because on average the stars have lower masses, and partly because there are fewer of them.

\section{ACKNOWLEDGEMENTS}

The authors like to thank the anonymous referee for the comments that helped to significantly improve the paper. PFR, SW, SDC, and AK acknowledge support via the European Research Council (ERC) starting grant No. 679852 'RADFEEDBACK'. DS and SW thank the Deutsche Forschungsgemeinschaft (DFG) for funding via the SFB 956 'Conditions \& impact of star formation', via the subprojects C5 and C6. APW gratefully acknowledges the support of a consolidated grant (ST/K00926/1) from the UK Science and Technology Facilities Council. The authors gratefully acknowledge the Gauss Centre for Supercomputing e.V. (www.gauss-centre.eu) for funding this project (ID: pr47pi) by providing computing time on the GCS Supercomputer SuperMUC at Leibniz Supercomputing Centre (http://www.lrz.de). PFR acknowledges D. Price for providing the visualization tool SPLASH (Price 2011).

\section{DATA AVAILABILITY}

The data underlying this article will be shared on reasonable request to the corresponding author.

\section{REFERENCES}

Aarseth S. J., 2003, Gravitational N-Body Simulations. Cambridge Univ. Press, Cambridge

Alves J., Lada C., Lada E., 2001, The Messenger, 103, 1

André P., Belloche A., Motte F., Peretto N., 2007, A\&A, 472, 519

André P., Di Francesco J., Ward-Thompson D., Inutsuka S. I., Pudritz R. E., Pineda J. E., 2014, in Beuther H., Klessen R. S., Dullemond C. P., Henning T., eds, Protostars and Planets VI, Univ. Arizona Press, Tucson, AZ, p. 27

André P. et al., 2010, A\&A, 518, L102

Arce H. G., Borkin M. A., Goodman A. A., Pineda J. E., Halle M. W., 2010, ApJ, 715, 1170

Arce H. G., Sargent A. I., 2006, ApJ, 646, 1070

Arce H. G., Shepherd D., Gueth F., Lee C.-F., Bachiller R., Rosen A., Beuther H., 2007, in Reipurth B., Jewitt D., Keil K., eds, Protostars and Planets V. Univ. Arizona Press, Tucson, AZ, 245

Bally J., 2016, ARA\&A, 54, 491

Bate M. R., 2005, MNRAS, 363, 363

Bate M. R., 2009a, MNRAS, 392, 1363

Bate M. R., 2009b, MNRAS, 397, 232

Bate M. R., 2012, MNRAS, 419, 3115

Bate M. R., Bonnell I. A., 2005, MNRAS, 356, 1201

Bate M. R., Bonnell I. A., Price N. M., 1995, MNRAS, 277, 362

Bate M. R., Tricco T. S., Price D. J., 2014, MNRAS, 437, 77

Bell K. R., Lin D. N. C., 1994, ApJ, 427, 987

Bjerkeli P., van der Wiel M. H. D., Harsono D., Ramsey J. P., Jørgensen J. K., 2016, Nature, 540, 406

Blandford R. D., Payne D. G., 1982, MNRAS, 199, 883

Bonnor W. B., 1956, Z. Astrophys., 39, 143

Calvet N., Hartmann L., Kenyon S. J., 1993, ApJ, 402, 623

Cesaroni R. et al., 2018, A\&A, 612, A103

Chabrier G., 2003, PASP, 115, 763

Chen X., Arce H. G., Zhang Q., Launhardt R., Henning T., 2016, ApJ, 824, 72

Chen X. et al., 2013, ApJ, 768, 110

Choi M. et al., 2017, ApJS, 232, 24

Commerçon B., Hennebelle P., Audit E., Chabrier G., Teyssier R., Henning T., 2012, in Capuzzo-Dolcetta R., Limongi M., Tornambè A., eds, ASP Conf. Ser. Vol. 453, Advances in Computational Astrophysics: Methods, Tools, and Outcome, Astron. Soc. Pac., San Francisco, p. 13

Connelley M. S., Reipurth B., Tokunaga A. T., 2008, AJ, 135, 2496

Croswell K., Hartmann L., Avrett E. H., 1987, ApJ, 312, 227

Cullen L., Dehnen W., 2010, MNRAS, 408, 669

Cunningham A. J., Klein R. I., Krumholz M. R., McKee C. F., 2011, ApJ, 740,107

Cunningham A. J., Krumholz M. R., McKee C. F., Klein R. I., 2018, MNRAS, 476, 771

Duchêne G., Bontemps S., Bouvier J., André P., Djupvik A. A., Ghez A. M., 2007, A\&A, 476, 229

Duchêne G., Kraus A., 2013, ARA\&A, 51, 269 
Duchêne G., Lacour S., Moraux E., Goodwin S., Bouvier J., 2018, MNRAS, 478,1825

Ebert R., 1957, ZA, 42, 263

El-Badry K., Rix H.-W., Tian H., Duchêne G., Moe M., 2019, MNRAS, 489, 5822

Feddersen J. R. et al., 2020, ApJ, 896, 11

Federrath C., Schrön M., Banerjee R., Klessen R. S., 2014, ApJ, 790, 128

Fernandez M. A. et al., 2017, PASP, 129, 084201

Frank A. et al., 2014, in Beuther H., Klessen R. S., Dullemond C. P., Henning T., eds, Protostars and Planets VI. Univ. Arizona Press, Tucson, AZ, p. 451

Friesen R. K. et al., 2017, ApJ, 843, 63

Goodwin S. P., Kroupa P., Goodman A., Burkert A., 2007, in Reipurth B., Jewitt D., Keil K., eds, Protostars and Planets V. Univ. Arizona Press, Tucson, AZ, p. 133

Gray W. J., McKee C. F., Klein R. I., 2018, MNRAS, 473, 2124

Guszejnov D., Hopkins P. F., Krumholz M. R., 2017, MNRAS, 468, 4093

Guszejnov D., Krumholz M. R., Hopkins P. F., 2016, MNRAS, 458, 673

Hansen C. E., Klein R. I., McKee C. F., Fisher R. T., 2012, ApJ, 747, 22

Hartigan P., Edwards S., Ghandour L., 1995, ApJ, 452, 736

Hartmann L., 1997, in Reipurth B., Bertout C., eds, IAU Symp. Vol. 182, Herbig-Haro Flows and the Birth of Stars, Kluwer, Dordrecht, p. 391

Hartmann L., Calvet N., 1995, AJ, 109, 1846

Hartmann L., Stauffer J. R., 1989, AJ, 97, 873

Hennebelle P., Commerçon B., Chabrier G., Marchand P., 2016, ApJ, 830, L8

Hennebelle P., Commerçon B., Joos M., Klessen R. S., Krumholz M., Tan J. C., Teyssier R., 2011, A\&A, 528, A72

Hennebelle P., Inutsuka S.-I., 2019, Front. Astron. Space Sci., 6, 5

Hirota T., Machida M. N., Matsushita Y., Motogi K., Matsumoto N., Kim M. K., Burns R. A., Honma M., 2017, Nat. Astron., 1, 0146

Holman K., Walch S. K., Goodwin S. P., Whitworth A. P., 2013, MNRAS, 432, 3534

Hubber D. A., Rosotti G. P., Booth R. A., 2018, MNRAS, 473, 1603

Hubber D. A., Walch S., Whitworth A. P., 2013, MNRAS, 430, 3261

Johnstone D., Wilson C. D., Moriarty-Schieven G., Joncas G., Smith G., Gregersen E., Fich M., 2000, ApJ, 545, 327

Jones M. O., Bate M. R., 2018, MNRAS, 480, 2562

Kandori R. et al., 2018, ApJ, 865, 121

Kim W.-T., Ostriker E. C., 2001, ApJ, 559, 70

Kounkel M. et al., 2019, AJ, 157, 196

Königl A., Pudritz R. E., 2000, in Mannings V., Boss A. P., Russell S. S., eds, Protostars and Planets IV, Univ. Arizona Press, Tucson, AZ, p. 759

Könyves V. et al., 2015, A\&A, 584, A91

Könyves V. et al., 2020, A\&A, 635, A34

Kroupa P., 2001, MNRAS, 322, 231

Kroupa P., 2002, Science, 295, 82

Krumholz M. R., Federrath C., 2019, Front. Astron. Space Sci., 6, 7

Krumholz M. R., Klein R. I., McKee C. F., 2012, ApJ, 754, 71

Krumholz M. R. et al., 2014, in Beuther H., Klessen R. S., Dullemond C. P., Henning T., eds, Protostars and Planets VI, Univ. Arizona Press, Tucson, AZ, p. 243

Kuffmeier M., Calcutt H., Kristensen L. E., 2019, A\&A, 628, A112

Kuiper R., Yorke H. W., Turner N. J., 2015, ApJ, 800, 86

Launhardt R. et al., 2009, A\&A, 494, 147

Lee C.-F., Ho P. T. P., Li Z.-Y., Hirano N., Zhang Q., Shang H., 2017, Nat. Astron., 1, 0152

Leroy A. K., Walter F., Brinks E., Bigiel F., de Blok W. J. G., Madore B., Thornley M. D., 2008, AJ, 136, 2782

Lewis B. T., Bate M. R., 2017, MNRAS, 467, 3324

Li P. S., Klein R. I., McKee C. F., 2018, MNRAS, 473, 4220

Liu J., Qiu K., Wyrowski F., Menten K., Guesten R., Cao Y., Wang Y., 2018, ApJ, 860, 106

Lomax O., Whitworth A. P., Hubber D. A., Stamatellos D., Walch S., 2014, MNRAS, 439, 3039

Lomax O., Whitworth A. P., Hubber D. A., Stamatellos D., Walch S., 2015, MNRAS, 447, 1550

Lombardi J. C., McInally W. G., Faber J. A., 2015, MNRAS, 447, 25
Louvet F., Dougados C., Cabrit S., Mardones D., Ménard F., Tabone B., Pinte C., Dent W. R. F., 2018, A\&A, 618, A120

Lucy L. B., 2006, A\&A, 457, 629

Lynden-Bell D., 2003, MNRAS, 341, 1360

Machida M. N., 2014, ApJ, 796, L17

Machida M. N., Basu S., 2019, ApJ, 876, 149

Machida M. N., Hosokawa T., 2013, MNRAS, 431, 1719

Machida M. N., Inutsuka S.-I., Matsumoto T., 2008, ApJ, 676, 1088

Machida M. N., Inutsuka S.-I., Matsumoto T., 2009, Astrophys. Space Sci. Proc., 13, 405

Marsh K. A. et al., 2016, MNRAS, 459, 342

Matsumoto T., Saigo K., Takakuwa S., 2019, ApJ, 871, 36

Matt S., Pudritz R. E., 2005, ApJ, 632, L135

Matzner C. D., McKee C. F., 1999, ApJ, 526, L109

Maury A. J. et al., 2019, A\&A, 621, A76

McKee C. F., Offner S. R. R., 2011, in Alves J., Elmegreen B. G., Girart J. M., Trimble V., eds, IAU Symposium, Vol. 270, Computational Star Formation. Kluwer, Dordrecht, p. 73

McKee C. F., Offner S. S. R., 2010, ApJ, 716, 167

Mercer A., Stamatellos D., 2017, MNRAS, 465, 2

Monaghan J. J., Lattanzio J. C., 1985, A\&A, 149, 135

Morris J. P., Monaghan J. J., 1997, J. Comput. Phys., 136, 41

Mundt R., Fried J. W., 1983, ApJ, 274, L83

Murray D., Goyal S., Chang P., 2018, MNRAS, 475, 1023

Murray N., 2011, ApJ, 729, 133

Myers A. T., Klein R. I., Krumholz M. R., McKee C. F., 2014, MNRAS, 439, 3420

Myers P. C., 2009, ApJ, 700, 1609

Nakamura F., Li Z.-Y., 2007, ApJ, 662, 395

Nakano T., Hasegawa T., Morino J.-I., Yamashita T., 2000, ApJ, 534, 976

Nakano T., Hasegawa T., Norman C., 1995, ApJ, 450, 183

Nisini B., Antoniucci S., Alcalá J. M., Giannini T., Manara C. F., Natta A., Fedele D., Biazzo K., 2018, A\&A, 609, A87

Offner S. S. R., 2011, in Alves J., Elmegreen B. G., Girart J. M., Trimble V., eds, IAU Symp. Vol. 270, Computational Star Formation. Kluwer, Dordrecht, p. 231

Offner S. S. R., Arce H. G., 2014, ApJ, 784, 61

Offner S. S. R., Chaban J., 2017, ApJ, 847, 104

Offner S. S. R., Klein R. I., McKee C. F., Krumholz M. R., 2009, ApJ, 703, 131

Pelletier G., Pudritz R. E., 1992, ApJ, 394, 117

Peters T., Klaassen P. D., Mac Low M.-M., Schrön M., Federrath C., Smith M. D., Klessen R. S., 2014, ApJ, 788, 14

Pineda J. E., Goodman A. A., Arce H. G., Caselli P., Longmore S., Corder S., 2011, ApJ, 739, L2

Pineda J. E. et al., 2015, Nature, 518, 213

Plunkett A. L., Arce H. G., Corder S. A., Mardones D., Sargent A. I., Schnee S. L., 2013, ApJ, 774, 22

Price D. J., 2011, SPLASH: An Interactive Visualization Tool for Smoothed Particle Hydrodynamics Simulations, Astrophysics Source Code Library, record ascl:1103.004

Price D. J., Tricco T. S., Bate M. R., 2012, MNRAS, 423, L45

Pudritz R. E., Ouyed R., Fendt C., Brandenburg A., 2007, in Reipurth B., Jewitt D., Keil K., eds, Protostars and Planets V. Univ. Arizona Press, Tucson, AZ, p.277

Raghavan D. et al., 2010, ApJS, 190, 1

Reipurth B., 1989, Nature, 340, 42

Reipurth B., Bally J., 2001, ARA\&A, 39, 403

Reipurth B., Zinnecker H., 1993, A\&A, 278, 81

Rice W. K. M., Armitage P. J., Mamatsashvili G. R., Lodato G., Clarke C. J., 2011, MNRAS, 418, 1356

Rohde P. F., Walch S., Seifried D., Whitworth A. P., Clarke S. D., Hubber D. A., 2019, MNRAS, 483, 2563

Saiki Y., Machida M. N., 2020, ApJ, 897, L22

Samal M. R., Chen W. P., Takami M., Jose J., Froebrich D., 2018, MNRAS, 477,4577

Schruba A., Kruijssen J. M. D., Leroy A. K., 2019, ApJ, 883, 2 
Seifried D., Banerjee R., Pudritz R. E., Klessen R. S., 2013, MNRAS, 432, 3320

Seifried D., Banerjee R., Pudritz R. E., Klessen R. S., 2015, MNRAS, 446, 2776

Seifried D., Pudritz R. E., Banerjee R., Duffin D., Klessen R. S., 2012 MNRAS, 422, 347

Shan Y. et al., 2017, ApJ, 846, 93

Shu F. H., Adams F. C., 1987, in Appenzeller I., Jordan C., eds, IAU Symp. Vol. 122, Circumstellar Matter. Kluwer, Dordrecht, p. 7

Shu F. H., Lizano S., Ruden S. P., Najita J., 1988, ApJ, 328, L19

Simon M., Obbie R. C., 2009, AJ, 137, 3442

Springel V., Hernquist L., 2002, MNRAS, 333, 649

Stamatellos D., Whitworth A. P., Bisbas T., Goodwin S., 2007, A\&A, 475, 37

Stamatellos D., Whitworth A. P., Hubber D. A., 2012, MNRAS, 427, 1182

Stephens M. A., 1974, J. Am. Stat. Assoc., 69, 730

Tabone B. et al., 2017, A\&A, 607, L6

Tafalla M., Myers P. C., Caselli P., Walmsley C. M., 2004, A\&A, 416, 191

Tafalla M., Santiago-García J., Hacar A., Bachiller R., 2010, A\&A, 522, A91

Tan J. C., McKee C. F., 2004, ApJ, 603, 383

Tobin J. J. et al., 2016, ApJ, 818, 73

Tobin J. J. et al., 2018, ApJ, 867, 43

Tokovinin A. A., 2000, A\&A, 360, 997

Tomida K., 2014, ApJ, 786, 98

Tomida K., Okuzumi S., Machida M. N., 2015, ApJ, 801, 117

Toomre A., 1964, ApJ, 139, 1217

Tout C. A., Pols O. R., Eggleton P. P., Han Z., 1996, MNRAS, 281, 257
Troland T. H., Crutcher R. M., 2008, ApJ, 680, 457

Utomo D. et al., 2018, ApJ, 861, L18

Valtonen M., Karttunen H., Gutzwiller M. C., 2007, Phys. Today, 60, 59

Walch S., Naab T., Whitworth A., Burkert A., Gritschneder M., 2010, MNRAS, 402, 2253

Walch S., Whitworth A. P., Girichidis P., 2012, MNRAS, 419, 760

Wang P., Li Z.-Y., Abel T., Nakamura F., 2010, ApJ, 709, 27

Whitworth A. P., Chapman S. J., Bhattal A. S., Disney M. J., Pongracic H., Turner J. A., 1995, MNRAS, 277, 727

Whitworth A. P., Lomax O., 2015, MNRAS, 448, 1761

Wurster J., Bate M. R., 2019, MNRAS, 486, 2587

Wurster J., Bate M. R., Price D. J., 2019, MNRAS, 489, 1719

Wurster J., Li Z.-Y., 2018, Front. Astron. Space Sci., 5, 39

Wurster J., Price D. J., Bate M. R., 2016, MNRAS, 457, 1037

Young M. D., Clarke C. J., 2015, MNRAS, 452, 3085

Zhang Y. et al., 2016, ApJ, 832, 158

Zhang Y. et al., 2018, ApJ, 864, 76

Zhang Y. et al., 2019, ApJ, 883, 1

Zhao B., Caselli P., Li Z.-Y., Krasnopolsky R., 2018, MNRAS, 473, 4868

Zhuchkov R. Y., Kiyaeva O. V., Orlov V. V., 2010, Astron. Rep., 54, 38

Zhu Z., Hartmann L., Gammie C., 2009, ApJ, 694, 1045

Zhu Z., Hartmann L., Gammie C. F., Book L. G., Simon J. B., Engelhard E., 2010, ApJ, 713, 1134

This paper has been typeset from a $\mathrm{T}_{\mathrm{E}} \mathrm{X} / \mathrm{LT} \mathrm{E} \mathrm{X}$ file prepared by the author. 